\title{
Information-entropic measures for non-zero $l$ states of confined hydrogen-like ions
}

\author{
Neetik Mukherjee* and Amlan K. Royt \\ Department of Chemical Sciences \\ Indian Institute of Science Education and Research (IISER) Kolkata, \\ Mohanpur-741246, Nadia, WB, India
}

\begin{abstract}
Rényi entropy $(R)$, Tsallis entropy $(T)$, Shannon entropy $(S)$, and Onicescu energy $(E)$ are studied in a spherically confined $\mathrm{H}$ atom (CHA), in conjugate space, with special emphasis on non-zero $l$ states. This work is a continuation of our recently published work [1]. Representative calculations are done by employing exact analytical wave functions in $r$ space. Accurate $p$ space-wave functions are generated numerically by performing Fourier transform on respective $r$-space counterparts. Further, these are extended for H-isoelectronic series by applying the scaling relations. $R, T$ are evaluated by choosing the order of entropic moments $(\alpha, \beta)$ as $\left(\frac{3}{5}, 3\right)$ in $r$ and $p$ spaces. Detailed, systematic results of all these measures with respect to variations of confinement radius $r_{c}$ are offered here for arbitrary $n, l$ quantum numbers. For a given $n$, at small $r_{c}, R_{\mathbf{r}}^{\alpha}, T_{\mathbf{r}}^{\alpha}, S_{\mathbf{r}}$ collapse with rise of $l$, attain a minimum, then again grow up. Growth in $r_{c}$ shifts the point of inflection towards higher $l$ values. An increase in $Z$ enhances localization of a particular state. Several other new interesting inferences are uncovered. Comparison with literature results (available only for $S$ in $2 p, 3 d$ states), offers excellent agreement.
\end{abstract}

PACS: 03.65-w, 03.65Ca, 03.65Ta, 03.65.Ge, 03.67-a.

Keywords: Rényi entropy, Shannon entropy, Onicescu energy, Tsallis entropy, Confined hydrogen atom, Scaling relation.

\footnotetext{
*Email: neetik.mukherjee@iiserkol.ac.in.

†Corresponding author. Email: akroy@iiserkol.ac.in, akroy6k@gmail.com.
} 


\section{INTRODUCTION}

Confinement of an atom or molecule inside an impenetrable cavity was first studied in the fourth decade of twentieth century [2]. Progress of research on such quantum systems was reviewed several times [2 -5] recording their importance in both fundamental physics and chemistry as well as in various engineering branches. They have relevance in many different physical situations, e.g., atoms under plasma environment, impurities in crystal lattice and semiconductor materials, trapping of atoms/molecules in zeolite cages or inside an endohedral cobweb of fullerenes, quantum wells, quantum wires, quantum dots [6] and so forth. Furthermore, such models were designed to mimic the high pressure environment inside the core of planets. Also, they have contemporary significance in interpreting various astrophysical phenomena [7] and many other interesting areas.

Theoretical study of a Hydrogen atom within an infinite spherical cavity was first published in 1937 [8]. Over the years, this simple confined hydrogen atom (CHA) model has served as a precursor to improve our understanding about the consequences of confinement in atomic electronic structure. In last decade, a CHA under the influence of various restricted environment has been extensively followed. Majority of these investigations include trapping of $\mathrm{H}$ atom either in a spherical box of penetrable, impenetrable walls or inside a hard box of different geometrical shape and size [5, 9-12]. In the realm of atomic physics, CHA provides us with many attractive physical and chemical properties. Numerous theoretical methods like perturbation theory, Padé approximation, WKB method, Hypervirial theorem, power-series solution, Lie algebra, Lagrange-mesh method, asymptotic iteration method, generalized pseudo-spectral (GPS) method were invoked for their proper treatment. Many interesting aspects such as rearrangement and redistribution of ground and excited energy states, simultaneous and incidental degeneracy, change in hyperfine splitting constant as well as dipole shielding factor, nuclear magnetic screening constant, pressure, variation of static and dynamic polarizability, hyperpolarizability, information entropy, etc., were probed with varying confining radius $\left(r_{c}\right)$. A vast literature exists on the subject; here we refer to a selective set [13-24]. Eigenvalues and eigenfunctions of CHA can be solved exactly in terms of Kummer M-function (confluent hypergeometric) [17].

In past twenty years, information measures were explored extensively for various quantum systems in both free and confinement situations. Some such potentials are: Pöschl-Teller 
[25], Rosen-Morse [26], pseudo-harmonic [27], squared tangent well [28], hyperbolic [29], position-dependent mass Schrödinger equation [30, 31], infinite circular well [32], hyperbolic double-well (DW) potential [33], etc. Recently, entropic measures were successfully engaged to understand trapping and oscillation of a particle within symmetric, asymmetric DW potential [34, 35], confined quantum harmonic oscillator [36], CHA [9, 10], etc.

Information-theoretic measures like Rényi entropy $(R)$, Tsallis entropy $(T)$, Shannon entropy $(S)$ and Onicescu energy $(E)$, in atomic systems may provide detailed knowledge about diffusion of atomic orbitals, spread of electron density, periodic properties, correlation energy and so forth [37-40]. R, T, called information generating functionals, are directly connected to entropic moments and completely quantify density. Former has been effectively employed to illustrate quantum entanglement, chemical reactivity, de-coherence and localization properties of Rydberg states of atoms [41-47]. Similarly, $T$ has been implicated specially for non-extensive thermo-statistics [48, 49] and gravitation [50, 51], etc. It is noteworthy that, $S, E$ are two special cases of $R, T$. Former measures extent of concentration of the system wave function in respective space, whereas latter symbolizes expectation values of density. $S$ has its application in illuminating colin conjecture, atomic avoided crossing, orbital free density functional theory [52 56] in many-electron systems, etc. Likewise, $E$ has been widely used to estimate correlation energy and first ionisation potential [40].

In past few years appreciable attention has been paid to explore $S$ in both $r$ and $p$ space for CHA under soft and hard confinement [9]. Very recently, $S$ in conjugate space has been examined (for low-lying $s, p, d$ orbitals) with the help of variation principle employing Slater type orbitals [10]. However, rest of the information measures like $R, T, E$ have been attempted very rarely, with the exception of some first few $s$-states of CHA [1]. Hence, our primary motivation is to undertake a detailed analysis of $R, T, S, E$ in a CHA-like system in a systematic fashion for an arbitrary state characterized by principal and azimuthal quantum numbers $n, l$, in both spaces, with special emphasis on $l \neq 0$. Illustrative calculations are performed with exact analytical wave functions in $r$-space; whereas in $p$ space, numerical wave functions are generated by executing Fourier transform on the eigenfunction of respective $r$-space orbitals. To put things in proper perspective, in this communication, $2 p, 3 d, 4 f, 5 g$ and $10 s-10 m$ states have been chosen as representatives. By considering all the acceptable l's corresponding to a given $n$, one can follow the changes in behavior of $l$ states as the environment switches from free to confinement. Such a comparative study 
of these information measures are done with respect to their free Hydrogen atom (FHA) counterpart. We also inspect the nature of $R, T, S, E$ for hydrogenic isoelectronic series (by varying atomic number $Z$ ) inside the spherical impenetrable cavity, using the scaling properties [57] satisfied by such a system. This time we restrict ourselves to ground state only; for extension to other states is straightforward. To this end, all measures in a CHA-like atoms are obtained in both $r, p$ and composite spaces. Note that such studies in a CHA are very rare and as already implied, most of the present results are offered here for the first time. Throughout the article, comparison with existing literature results are made wherever possible. Organization of this article is as follows. Section II gives a brief account of the theoretical method used; Sec. III presents a detailed discussion on the results of $R, T, S, E$ of CHA and H-isoelectronic series, while we conclude with a few remarks in Sec. IV.

\section{THEORETICAL METHOD}

The time-independent, non-relativistic radial Schrödinger equation under the influence of confinement, without loss of generality, for a central potential, may be written as,

$$
\left[-\frac{1}{2} \frac{d^{2}}{d r^{2}}+\frac{\ell(\ell+1)}{2 r^{2}}+v(r)+v_{c}(r)\right] \psi_{n, \ell}(r)=\mathcal{E}_{n, \ell} \psi_{n, \ell}(r)
$$

where $v(r)=-\frac{Z}{r}$ ( $Z$ implies atomic number $)$. Our desired effect of radial confinement inside an impenetrable hard cavity can be modeled by invoking the following form of potential: $v_{c}(r)=+\infty$ for $r>r_{c}$ and 0 for $r \leq r_{c}$, where $r_{c}$ corresponds to radius of the cage. It is worthwhile mentioning that, atomic units are employed through out the calculations and $\mathbf{r}, \mathbf{p}$ subscripts denote quantities in full $r$ and $p$ spaces (including the angular part) respectively.

On solving Eq. (1) one can obtain following exact form for eigenfunctions in CHA [17],

$\psi_{n, l}(Z r)=N_{n, l}\left(2 Z r \sqrt{-2 \mathcal{E}_{n, l}}\right)^{l}{ }_{1} F_{1}\left[\left(l+1-\frac{1}{\sqrt{-2 \mathcal{E}_{n, l}}}\right),(2 l+2), 2 Z r \sqrt{-2 \mathcal{E}_{n, l}}\right] e^{-Z r \sqrt{-2 \mathcal{E}_{n, l}}}$.

Here, $N_{n, l}$ represents the normalization constant, $\mathcal{E}_{n, l}$ prevails to energy of a given $n, l$ state while ${ }_{1} F_{1}[a, b, r]$ refers to confluent hypergeometric function. Allowed energies at a given $r_{c}$ can be retrieved by applying the Dirichlet boundary condition that $\psi_{n, \ell}(0)=\psi_{n, \ell}\left(r_{c}\right)=0$ and finding the zeros of ${ }_{1} F_{1}$, such that,

$$
{ }_{1} F_{1}\left[\left(l+1-\frac{1}{\sqrt{-2 \mathcal{E}_{n, l}}}\right),(2 l+2), 2 Z r_{c} \sqrt{-2 \mathcal{E}_{n, l}}\right]=0 .
$$


For a particular $l$, first root signifies energy of the lowest state having $\left(n_{\text {lowest }}=l+1\right)$, and consecutive roots imply excited states. Note that, for construction of exact wave function of CHA for a specific state, one needs to provide the energy eigenvalue of that state. In our present calculation, $\mathcal{E}_{n, l}$ of CHA are computed by invoking the GPS [23] method. This is applied, because in this pursuit, we are interested in the information measures in CHA, for which GPS energies have been found to be sufficiently accurate to provide correct eigenvalues and eigenfunctions. Over the years, this has been tested in a varied case of important model and realistic potentials, including both free and confinement situations [23, 58 60]. Also, it is obvious from Eq. (2) that, $\mathcal{E}_{n, l}$ depends on the product $Z r_{c}$. Hence in spite of changes in $Z$ and $r_{c}$ separately, if their product remains constant, then $\mathcal{E}_{n, l}$ will not be affected.

The angular part has following common form in both $r$ and $p$ spaces $\left(P_{l}^{m}(\cos \theta)\right.$ signifies usual associated Legendre polynomial),

$$
Y_{l, m}(\Omega)=\Theta_{l, m}(\theta) \Phi_{m}(\phi)=(-1)^{m} \sqrt{\frac{2 l+1}{4 \pi} \frac{(l-m) !}{(l+m) !}} P_{l}^{m}(\cos \theta) e^{-i m \phi} .
$$

The $p$-space wave function $(\mathbf{p}=\{p, \Omega\})$ for a particle in a central potential is obtained from respective Fourier transform of its $r$-space counterpart, and as such, is given below,

$$
\begin{aligned}
\psi_{n, l}(p) & =\frac{1}{(2 \pi)^{\frac{3}{2}}} \int_{0}^{r_{c}} \int_{0}^{\pi} \int_{0}^{2 \pi} \psi_{n, l}(r) \Theta(\theta) \Phi(\phi) e^{i p r \cos \theta} r^{2} \sin \theta \mathrm{d} r \mathrm{~d} \theta \mathrm{d} \phi, \\
& =\frac{1}{2 \pi} \sqrt{\frac{2 l+1}{2}} \int_{0}^{r_{c}} \int_{0}^{\pi} \psi_{n, l}(r) P_{l}^{0}(\cos \theta) e^{i p r \cos \theta} r^{2} \sin \theta \mathrm{d} r \mathrm{~d} \theta .
\end{aligned}
$$

Note that, here $\psi(p)_{n, l}$ needs to be normalized. Integrating over $\theta$ and $\phi$ variables, Eq. (5) may be further rewritten as given below,

$$
\psi_{n, l}(p)=(-i)^{l} \int_{0}^{r_{c}} \frac{\psi_{n, l}(r)}{p} f(r, p) \mathrm{d} r .
$$

Depending on $l, f(r, p)$ can be expressed in following simplified form ( $m^{\prime}$ starts with 0$)$,

$$
\begin{aligned}
& f(r, p)=\quad \sum_{k=2 m^{\prime}+1}^{m^{\prime}<\frac{l}{2}} a_{k} \frac{\cos p r}{p^{k} r^{k-1}}+\sum_{j=2 m^{\prime}}^{m^{\prime}=\frac{l}{2}} b_{j} \frac{\sin p r}{p^{j} r^{j-1}}, \quad \text { for even } l, \\
& f(r, p)=\sum_{k=2 m^{\prime}}^{m^{\prime}=\frac{l-1}{2}} a_{k} \frac{\cos p r}{p^{k} r^{k-1}}+\sum_{j=2 m^{\prime}+1}^{m^{\prime}=\frac{l-1}{2}} b_{j} \frac{\sin p r}{p^{j} \text { rerst } \operatorname{s}_{c} a_{6} \cdot \text { tex }^{j-1}}, \quad \text { for odd } l .
\end{aligned}
$$

The coefficients $a_{k}, b_{j}$ of even- $l$ and odd- $l$ states are calculated using Eq. (5). 
Let $\rho(\mathbf{r})$ and $\Pi(\mathbf{p})$ denote normalized position and momentum electron densities for CHA. Then, position, momentum shannon entropies $\left(S_{\mathbf{r}}, S_{\mathbf{p}}\right)$ and their sum $\left(S_{t}\right)$ for H-like atoms are defined in terms of expectation values of logarithmic probability density functions and $Z$, which for a central potential further simplify to,

$$
\begin{aligned}
S_{\mathbf{r}}(Z) & =-3 \ln Z+S_{\mathbf{r}}(Z=1), \quad S_{\mathbf{p}}(Z)=3 \ln Z+S_{\mathbf{p}}(Z=1) \\
S_{\mathbf{r}}(Z=1) & =-\int_{\mathcal{R}^{3}} \rho(\mathbf{r}) \ln [\rho(\mathbf{r})] \mathrm{d} \mathbf{r}, \quad S_{\mathbf{p}}(Z=1)=-\int_{\mathcal{R}^{3}} \Pi(\mathbf{p}) \ln [\Pi(\mathbf{p})] \mathrm{d} \mathbf{p} \\
S_{t} & =S_{\mathbf{r}}(Z)+S_{\mathbf{p}}(Z)=S_{\mathbf{r}}(Z=1)+S_{\mathbf{p}}(Z=1)
\end{aligned}
$$

The above equation clearly suggests that, at a fixed $r_{c}$, both $S_{\mathbf{r}}(Z)$ and $S_{\mathbf{p}}(Z)$ are linear functions of logarithm of $Z$ with slope -3 and 3 respectively. Moreover, $S_{\mathbf{r}}(Z=1), S_{\mathbf{p}}(Z=$ 1 ) act as intercepts in $r$ - and $p$-space equations. The last equation implies that addition of $S_{\mathbf{r}}(Z)$ and $S_{\mathbf{p}}(Z)$ produces same result as one obtains from the corresponding sum for $Z=1$. Hence $S_{t}$ will remain unaltered with change in $Z$. Actually it has been established [57] that it solely depends on $Z r_{c}$ product as evident from Eq. (3), rather than individual $Z$ and $r_{c}$. Here and in the discussion throughout the article, $Z$ dependence is identified in the parentheses of all respective measures, for $Z>1$. For CHA $(Z=1)$, there are no parentheses in the expressions of $R, T, S, E$.

Similarly, Rényi entropies of order $\lambda(\neq 1)$ are obtained by taking logarithm of $\lambda$-order entropic moment. In spherical polar coordinate, they are expressed as,

$$
\begin{aligned}
R_{\mathbf{r}}^{\lambda}(Z=1)=\frac{1}{1-\lambda} \ln \left(\int_{\mathcal{R}^{3}} \rho^{\lambda}(\mathbf{r}) \mathrm{d} \mathbf{r}\right) & =\frac{1}{(1-\lambda)} \ln \left(2 \pi \int_{0}^{\infty}[\rho(r)]^{\lambda} r^{2} \mathrm{~d} r \int_{0}^{\pi}[\chi(\theta)]^{\lambda} \sin \theta \mathrm{d} \theta\right) \\
& =\frac{1}{(1-\lambda)}\left(\ln 2 \pi+\ln \left[\omega_{r}^{\lambda}\right]+\ln \left[\omega_{(\theta, \phi)}^{\lambda}\right]\right), \\
R_{\mathbf{p}}^{\lambda}(Z=1)=\frac{1}{1-\lambda} \ln \left[\int_{\mathcal{R}^{3}} \Pi^{\lambda}(\mathbf{p}) \mathrm{d} \mathbf{p}\right] & =\frac{1}{(1-\lambda)} \ln \left(2 \pi \int_{0}^{\infty}[\Pi(p)]^{\lambda} p^{2} \mathrm{~d} p \int_{0}^{\pi}[\chi(\theta)]^{\lambda} \sin \theta \mathrm{d} \theta\right) \\
& =\frac{1}{(1-\lambda)}\left(\ln 2 \pi+\ln \left[\omega_{p}^{\lambda}\right]+\ln \left[\omega_{(\theta, \phi)}^{\lambda}\right]\right) .
\end{aligned}
$$

where $\omega_{\tau}^{\lambda}$ 's are entropic moments in $\tau(r$ or $p$ or $\theta, \phi)$ space with order $\lambda$, having forms,

$$
\omega_{r}^{\lambda}=\int_{0}^{\infty}[\rho(r)]^{\lambda} r^{2} \mathrm{~d} r, \quad \text { erst } h a_{6} \cdot \operatorname{tex} \omega_{p}^{\lambda}=\int_{0}^{\infty}[\Pi(p)]^{\lambda} p^{2} \mathrm{~d} p, \quad \omega_{(\theta, \phi)}^{\lambda}=\int_{0}^{\pi}[\chi(\theta)]^{\lambda} \sin \theta \mathrm{d} \theta .
$$

Here if $\lambda$ corresponds to $\alpha, \beta$ in $r, p$ spaces respectively, then they are related as $\frac{1}{\alpha}+\frac{1}{\beta}=2$. 
In that case, one can define Rényi entropy sum as,

$$
R_{t}^{(\alpha, \beta)}=\frac{2-\alpha-\beta}{(1-\alpha)(1-\beta)} \ln 2 \pi+\frac{1}{(1-\alpha)}\left(\ln \left[\omega_{r}^{\alpha}\right]+\ln \left[\omega_{(\theta, \phi)}^{\alpha}\right]\right)+\frac{1}{(1-\beta)}\left(\ln \left[\omega_{p}^{\beta}\right]+\ln \left[\omega_{(\theta, \phi)}^{\beta}\right]\right) .
$$

Equation (9) suggests that, at a particular $r_{c}$, like $S_{\mathbf{r}}(Z), S_{\mathbf{p}}(Z)$, both $R_{\mathbf{r}}^{\lambda}(Z)$ and $R_{\mathbf{p}}^{\lambda}(Z)$ also linearly depend on $\ln Z$ (slope $-3,3$ respectively) with intercepts $R_{\mathbf{r}}^{\lambda}(Z=1)$ and $R_{\mathbf{p}}^{\lambda}(Z=1)$ respectively. Further, as with $S_{t}, R^{(\alpha, \beta)}$ also remains unimpacted with change of $Z$.

In a similar fashion, Tsallis entropies [61] in $r, p$ space and their product can be written down as below,

$$
\begin{gathered}
T_{\mathbf{r}}^{\alpha}(Z)=\left(\frac{1}{\alpha-1}\right)\left[1-\frac{1}{Z^{3}} \int_{\mathcal{R}^{3}} \rho^{\alpha}(\mathbf{r}) \mathrm{d} \mathbf{r}\right]=\left(\frac{1}{\alpha-1}\right)\left[1-\frac{1}{Z^{3}} \omega_{r}^{\alpha} \omega_{(\theta, \phi)}^{\alpha}\right] \\
T_{\mathbf{p}}^{\beta}(Z)=\left(\frac{1}{\beta-1}\right)\left[1-Z^{3} \int_{\mathcal{R}^{3}} \Pi^{\beta}(\mathbf{p}) \mathrm{d} \mathbf{p}\right]=\left(\frac{1}{\beta-1}\right)\left[1-Z^{3} \omega_{r}^{\beta} \omega_{(\theta, \phi)}^{\beta}\right] \\
T_{t}^{(\alpha, \beta)}(Z)=T_{\mathbf{r}}^{\alpha}(Z) T_{\mathbf{p}}^{\beta}(Z)
\end{gathered}
$$

One sees that, $T_{\mathbf{r}}^{\alpha}(Z)$ reduces and $T_{\mathbf{p}}^{\beta}(Z)$ enhances with rise of $Z$. Note that $\alpha=\beta=2$ leads to Onicescu energy $E$, which in $r$ and $p$ spaces are given as,

$$
\begin{array}{r}
E_{\mathbf{r}}(Z)=Z^{3} \omega_{r}^{2} \omega_{(\theta, \phi)}^{2}=Z^{3} E_{\mathbf{r}}(Z=1) \\
E_{\mathbf{p}}(Z)=\frac{1}{Z^{3}} \omega_{r}^{2} \omega_{(\theta, \phi)}^{2}=\frac{1}{Z^{3}} E_{\mathbf{p}}(Z=1) \\
E_{t}(Z)=E_{\mathbf{r}}(Z) E_{\mathbf{p}}(Z)=E_{\mathbf{r}}(Z=1) E_{\mathbf{p}}(Z=1) .
\end{array}
$$

Here, $E_{t}$ represents onicescu energy product. Dependence of $E_{\mathbf{r}}(Z)$ and $E_{\mathbf{p}}(Z)$ on $Z$ is seen to be opposite to that of the previous three measures discussed above. Thus $E_{\mathbf{r}}(Z)$ grows up and $E_{\mathbf{p}}(Z)$ falls off with rise of $Z$. But, as usual, $E_{t}(Z)$ remains unchanged as $Z$ modifies.

\section{RESULT AND DISCUSSION}

At the outset, it is appropriate to mention a few things regarding the presentation. Here, our focus is to uncover the impact of an impenetrable spherical cage on non-zero $l$ states of CHA by using information-theoretic measures. The net information measures in conjugate $r$ and $p$ space of CHA may be segmented into two separate contributions, viz., (i) a radial and (ii) an angular part. In CHA, the radial barrier changes from infinity to a finite region without affecting angular boundary conditions. So angular portion remains invariant in $r$ and $p$ spaces; moreover they will also not change with respect to boundary 
TABLE I: Rényi entropies, $R_{\mathbf{r}}^{\alpha}, R_{\mathbf{p}}^{\beta}$ and $R_{t}^{(\alpha, \beta)}$ for $2 p, 3 d$ states of CHA at selected $r_{c}$ values, with $\alpha=\frac{3}{5}$ and $\beta=3$. See text for details.

\begin{tabular}{llll|llll}
\hline \hline$r_{c}$ & $R_{\mathbf{r}}^{\alpha}$ & $R_{\mathbf{p}}^{\beta}$ & $R t^{(\alpha, \beta)}$ & $r_{c}$ & $R_{\mathbf{r}}^{\alpha}$ & $R_{\mathbf{p}}^{\beta}$ & $3 d^{\boldsymbol{\Phi}}$ \\
\hline & & & & & $R_{t}^{(\alpha, \beta)}$ \\
\hline 0.1 & -6.16888358521 & 12.8086549 & 6.6397714 & 0.1 & -6.11864461237 & 13.2299266 & 7.111282 \\
0.2 & -4.09121892459 & 10.7314035 & 6.6401846 & 0.2 & -4.03973495813 & 11.1514674 & 7.1117325 \\
0.3 & -2.87663035551 & 9.5172358 & 6.6406055 & 0.3 & -2.82387786690 & 9.9360644 & 7.1121866 \\
0.5 & -1.34785892643 & 7.9893299 & 6.641471 & 0.5 & -1.29249680384 & 8.4056025 & 7.1131057 \\
0.8 & 0.05635500373 & 6.5864754 & 6.6428304 & 0.8 & 0.11582086058 & 6.9986916 & 7.1145125 \\
1 & 0.72175456102 & 5.9220245 & 6.643779 & 1 & 0.78408877031 & 6.3313805 & 7.1154693 \\
5 & 5.43150144784 & 1.2403914 & 6.6718928 & 7.5 & 6.77107758150 & 0.3857834 & 7.1568610 \\
15 & 7.78024380659 & -0.924233 & 6.8560108 & 15 & 8.69500260562 & -1.45238070 & 7.2426219 \\
25 & 7.92394945000 & -0.9742317 & 6.9497178 & 50 & 9.92580433711 & -2.311277606 & 7.614526731 \\
45 & 7.92577664624 & -0.9736503 & 6.9521263 & 100 & 9.92600859049 & -2.311283603 & 7.614724988 \\
\hline \hline
\end{tabular}

${ }^{!} R_{\mathbf{r}}^{\alpha}, R_{\mathbf{p}}^{\beta}, R_{t}^{(\alpha, \beta)}$ in FHA for $2 p$ states $(|m|=0)$ are: $7.925776675482,-0.9736503771629,6.952126298319$.

${ }^{\boldsymbol{I}} R_{\mathbf{r}}^{\alpha}, R_{\mathbf{p}}^{\beta}, R_{t}^{(\alpha, \beta)}$ in FHA for $3 d$ states $(|m|=0)$ are: $9.926008594642,-2.311283609195,7.614724985446$.

condition in $r_{c}$ in a CHA. However, they will be affected by $l, m$ quantum numbers. In current calculation, we have kept magnetic quantum number $m$ fixed at 0 , unless stated otherwise. It is clear from Eq. (2) that, in $r$ space, radial wave functions are available in closed analytical form. In $p$ space, numerical wave functions are achieved by employing Fourier transform on respective $r$-space eigenfunctions. All our results provided in tables and figures are computed numerically. It is expected that, a gradual increase in $r_{c}$ should lead to a delocalization in the system in such a fashion that, when $r_{c} \rightarrow \infty$, it should unfold to FHA. On the contrary, when $r_{c} \rightarrow 0$, impression of confinement is maximum. Thus, it is convenient to explore our analysis by choosing some specific $r_{c}$ values in the range of 0.1 to 100. This parametric rise in $r_{c}$ reveals evolution of system from maximum confinement to a free system. It may be remarked that, a detailed systematic analysis of these measures in low-lying $s$ states, along the lines of current work, has been initiated by present authors and will be published elsewhere [1].

Our presentation strategy is as follows. Initially, $2 p, 3 d, 4 f, 5 g$ states are selected for analysis of various information measures; they all individually represent the lowest (nodeless) state of respective $l$. This will help us follow changes in $R, S, T, E$ with respect to alterations in $l$. Additionally we also explore all the $l$ states corresponding to a given $n$ (here chosen 
TABLE II: $R_{\mathbf{r}}^{\alpha}$ and $R_{\mathbf{p}}^{\beta}$ for $10 l$ states of $\mathrm{CHA}$ at seven different $r_{c}$ values, namely $0.1,0.5,1,10,40,80,100$. Here, $\alpha, \beta$ are chosen to be $\frac{3}{5}$ and 3 respectively. See text for details.

\begin{tabular}{|c|c|c|c|c|c|c|c|}
\hline \multicolumn{8}{|c|}{$R_{\mathrm{r}}^{\alpha}$} \\
\hline$l$ & $r_{c}=0.1$ & $r_{c}=0.5$ & $r_{c}=1$ & $r_{c}=10$ & $r_{c}=40$ & $r_{c}=80$ & $r_{c}=100$ \\
\hline 0 & -6.0792476535 & -1.2500151671 & 0.8305932083 & 7.7616144459 & 11.9866555713 & 14.1264898722 & 14.8227228363 \\
\hline 1 & -6.3891801965 & -1.5604409325 & 0.5195411162 & 7.4386341260 & 11.6485744296 & 13.7938994865 & 14.4924250904 \\
\hline 2 & -6.3886059693 & -1.5600883216 & 0.5196127247 & 7.4330589592 & 11.6242072771 & 13.7633595572 & 14.4630426402 \\
\hline 3 & -6.3665110491 & -1.5381245479 & 0.5414121420 & 7.4515237538 & 11.6283879357 & 13.7541478868 & 14.4519363360 \\
\hline 4 & -6.3359608527 & -1.5076548776 & 0.5717793565 & 7.4797957142 & 11.6465100421 & 13.7573382983 & 14.4505385080 \\
\hline 5 & -6.2994173707 & -1.4711571510 & 0.6082191748 & 7.5150095855 & 11.6749072268 & 13.7721369041 & 14.4592392391 \\
\hline 6 & -6.2581496967 & -1.4299022978 & 0.6494567192 & 7.5558021952 & 11.7119190030 & 13.7982974268 & 14.4788958301 \\
\hline 7 & -6.2143631650 & -1.3860901364 & 0.6933001405 & 7.6001063439 & 11.7558856869 & 13.8354493928 & 14.5099982856 \\
\hline 8 & -6.1748354691 & -1.3464778435 & 0.7330180632 & 7.6416699182 & 11.8023199187 & 13.8818610231 & 14.5518827831 \\
\hline 9 & -6.1702611858 & -1.3416902256 & 0.7380727967 & 7.6515945538 & 11.8288561033 & 13.9252706635 & 14.5938547362 \\
\hline \multicolumn{8}{|c|}{$R_{\mathbf{p}}^{\beta}$} \\
\hline 0 & 17.764042 & 12.938472 & 10.863484 & 4.225406 & -0.104732 & -2.194616 & -3.015142 \\
\hline 1 & 16.990848 & 12.164511 & 10.087976 & 3.319124 & -0.643120 & -2.666435 & -3.423219 \\
\hline 2 & 16.609713 & 11.782989 & 9.705776 & 2.886201 & -0.749884 & -2.917930 & -3.674227 \\
\hline 3 & 16.31713 & 11.490168 & 9.412558 & 2.566775 & -1.040808 & -3.221412 & -3.822730 \\
\hline 4 & 16.059368 & 11.2322271 & 9.154340 & 2.292588 & -1.439844 & -3.385452 & -4.054590 \\
\hline 5 & 15.8154003 & 10.9881122 & 8.910008 & 2.037463 & -1.820272 & -3.487284 & -4.250016 \\
\hline 6 & 15.573099 & 10.745675 & 8.667378 & 1.786891 & -2.166955 & -3.735834 & -4.383047 \\
\hline 7 & 15.322708 & 10.495141 & 8.416651 & 1.529727 & -2.494875 & -4.1772092 & -4.645285 \\
\hline 8 & 15.052818 & 10.225072 & 8.146348 & 1.253299 & -2.824948 & -4.672206 & -5.156688 \\
\hline 9 & 14.7302406 & 9.9021894 & 7.823079 & 0.9218974 & -3.204373 & -5.196718 & -5.787983 \\
\hline
\end{tabular}

10) to understand the outcome as the count of radial nodes vary. Actually, for a given $l$, an increase in $n$ enhances spreading as well as number of radial nodes, whereas an increment in $l$ within in a given $n$ reduces radial node.

To begin with, Table I displays our calculated $R_{\mathbf{r}}^{\alpha}, R_{\mathbf{r}}^{\alpha}, R_{t}^{(\alpha, \beta)}$ for $2 p, 3 d$ states of CHA at a selected set of $r_{c}$; which differ from state to state. Similarly, Table S1 in supplementary material (SM) portrays all the above quantities as a function of $r_{c}$ for $4 f, 5 g$ states. In this and all following tables related to these four states, information quantities are provided at same set including ten $r_{c}$ 's. In all occasions, $R_{\mathbf{r}}^{\alpha}$ 's progress continuously with $r_{c}$ and finally converge to respective FHA behavior after some larger finite $r_{c}$. Interestingly, for all states, $R_{\mathbf{r}}^{\alpha}$ remain negative up to $r_{c}=0.5$, changing sign after that. In contrast, $R_{\mathbf{p}}^{\beta}$ 's, tend to diminish with rise of $r_{c}$; eventually they also merge to FHA (negative for all) in the end. As a combination of these two effects, $R^{(\alpha, \beta)}$ 's steadily grow with progress of $r_{c}$ and as usual 

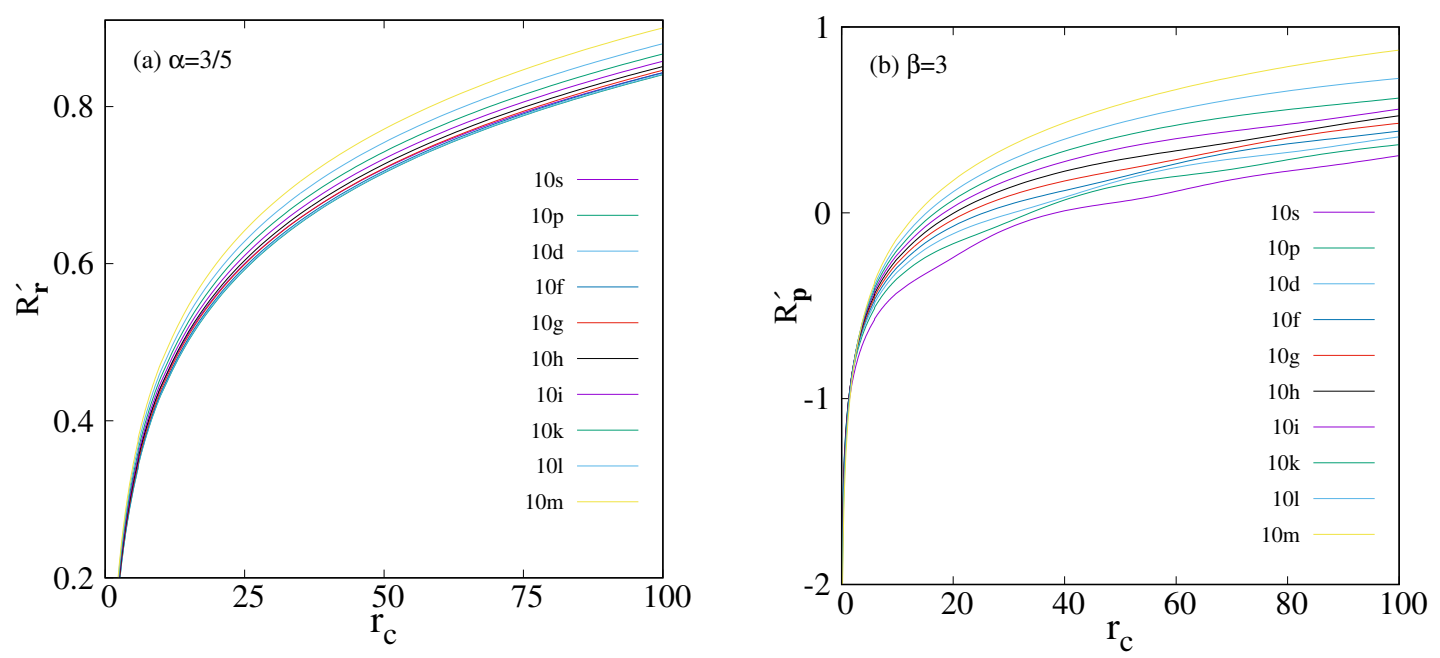

FIG. 1: Variation of $R_{\mathbf{r}}^{\prime}, R_{\mathbf{p}}^{\prime}$ with $r_{c}$ for $10 s-10 m$ states of CHA in panels (a), (b). $\alpha, \beta$ are chosen to be $\frac{3}{5}$ and 3 respectively. See text for detail.

they coalesce to respective borderline values finally. It is worth mentioning that, at small $r_{c}$, Rényi entropies in $r, p$ space obey same order: $R_{\mathbf{r}}^{\alpha}(5 g)>R_{\mathbf{r}}^{\alpha}(4 f)>R_{\mathbf{r}}^{\alpha}(3 d)>R_{\mathbf{r}}^{\alpha}(2 p)$ and $R_{\mathbf{p}}^{\beta}(5 g)>R_{\mathbf{p}}^{\beta}(4 f)>R_{\mathbf{p}}^{\beta}(3 d)>R_{\mathbf{p}}^{\beta}(2 p)$. But, at $r_{c} \rightarrow \infty$ limit, ordering in $p$ space completely reverses, while the $r$-space ordering is retained. The distribution pattern in $r$ space is observed presumably because, an increase in both $n, l$ leads to more delocalization of CHA. Next Table II provides $R_{\mathbf{r}}^{\alpha}, R_{\mathbf{p}}^{\beta}$ for all states having $l=0-9$, within $n=10$ of CHA, at seven selected $r_{c}$, namely $0.1,0.5,1,10,40,80,100$, featuring strong to medium confinement. At smaller $r_{c}(<1)$ region, $R_{\mathbf{r}}^{\alpha}$ s remain $(-)$ ve for all ten $l$; for rest five $r_{c}$ $(>1)$, the sign reverses. Numerical values surge with $r_{c}$ for all $l$. In all seven instances of $r_{c}, R_{\mathrm{r}}^{\alpha}$ 's initially fall as $l$ increments, then attain a minimum for some $l$ and again go up. In first three $r_{c}(0.1,0.5,1)$ these minima occur at $l=1$. At $r_{c}=10,40$ these happen at $l=2$. Further, These minima shift to $l=3$ and $l=4$ for $r_{c}=80$ and 100 respectively. In contrast, numerical values of $R_{\mathbf{p}}^{\beta}$ gradually lower with growth of $r_{c}$ for each $l$. Furthermore, in all seven $r_{c}$, it falls down with $l$ without going through any inflection points unlike its corresponding $r$-space counterpart. To the best of our knowledge, no such results have been reported for Rényi entropy in CHA. Hence they could not be directly compared, and hopefully would provide useful guidelines for future work.

Above changes of Table II are depicted in Fig. 1 in the form of ratios $R_{\mathbf{r}}^{\prime}=\left(\frac{R_{r}^{\alpha}(C H A)}{R_{\mathbf{r}}^{\alpha}(F H A)}\right)$ and $R_{\mathbf{p}}^{\prime}=\left(\frac{R_{p}^{\beta}(C H A)}{R_{\mathbf{p}}^{\beta}(F H A)}\right)$ for all $l$ states of $n=10$. For convenience, Rényi entropies of CHA, in both spaces, in this occasion, are divided by their respective FHA values. First it is 
TABLE III: Tsallis entropies $T_{\mathbf{r}}^{\alpha}, T_{\mathbf{p}}^{\beta}$ and $T^{(\alpha, \beta)}$ for $2 p, 3 d$ states of CHA at various $r_{c}$, for $\alpha, \beta$ as $\frac{3}{5}$ and 3 respectively. For more details, see text.

\begin{tabular}{llll|llll}
\hline \hline$r_{c}$ & $T_{\mathbf{r}}^{\alpha}$ & $T_{\mathbf{p}}^{\beta}$ & $T_{t}^{(\alpha, \beta)}$ & $r_{c}$ & $T_{\mathbf{r}}^{\alpha}$ & $T_{\mathbf{p}}^{\beta}$ & $T_{t}^{(\alpha, \beta)}$ \\
\hline & & $2 p^{!}$ & & & & $3 d^{\mathbf{q}}$ & \\
\hline 0.1 & -2.2880198630 & 0.4999999999 & -1.1440099313 & 0.1 & -2.28371690687 & 0.499999999998 & -1.1418584534 \\
0.2 & -2.0133435459 & 0.4999999997 & -1.0066717723 & 0.2 & -2.00321763758 & 0.499999999896 & -1.0016088186 \\
0.3 & -1.7089241387 & 0.4999999972 & -0.8544620646 & 0.3 & -1.69205429127 & 0.499999998828 & -0.8460271437 \\
0.5 & -1.0418811277 & 0.4999999425 & -0.5209405039 & 0.5 & -1.00923112502 & 0.499999974999 & -0.5046155373 \\
0.8 & 0.0569949807 & 0.4999990493 & 0.0284974362 & 0.8 & 0.11854567062 & 0.499999583146 & 0.0592727859 \\
1 & 0.8367342402 & 0.4999964094 & 0.4183641157 & 1 & 0.92097719569 & 0.499998416555 & 0.4604871395 \\
5 & 19.452725733 & 0.4581611514 & 8.9124832214 & 7.5 & 35.0142839008 & 0.268855906272 & 9.4137970306 \\
15 & 53.670306013 & -2.6750355 & -143.5699738 & 15 & 78.4872531785 & -8.630443042 & -677.379768 \\
25 & 56.993705192 & -3.0089477 & -171.4910836 & 50 & 130.0039513800 & -50.37685125 & -6549.18972146 \\
45 & 57.037203756 & -3.0048699 & -171.38938035 & 100 & 130.0147775743 & -50.37746147 & -6549.81444873 \\
\hline \hline
\end{tabular}

$! T_{\mathbf{r}}^{\alpha}, T_{\mathbf{p}}^{\beta}, T^{(\alpha, \beta)}$ in FHA for $2 p$ states $(|m|=0)$ are: $57.037204453034,-3.0048705041661,-171.38941330101483$.

${ }^{\top} T_{\mathbf{r}}^{\alpha}, T_{\mathbf{p}}^{\beta}, T^{(\alpha, \beta)}$ in FHA for $3 d$ states $(|m|=0)$ are: $130.014775913339,-50.377462107980,-6549.814447051782$.

relevant to recall the following facts about these two relative quantities. For any given $n, l$ $R_{\mathbf{r}}^{\alpha}$ enhances and $R_{\mathbf{p}}^{\beta}$ declines with rise of $r_{c}$, whereas for a FHA, they assume maximum and minimum values. Further, this minimum in the latter has negative sign. Such a division by their respective free counterpart makes these quantities unit-less and keeps upper bound to unity. This facilitates to observe a similar trend for two conjugate measures as $r_{c}$ varies. Simultaneous escalation of both the ratios with increment of $r_{c}$ suggests delocalization in the system. Further, unlike Table II, there is hardly any crossover among these $R_{\mathbf{r}}^{\prime}$ and $R_{\mathbf{p}}^{\prime}$ of $10 l$ states (there were crossovers in $R_{\mathrm{r}}^{\alpha}$ in Table II). More importantly, throughout the entire range of $r_{c}$, two ratios reduce with growth in $l$. This apparently indicates that states with greater number of nodes, experience the effects of confinement to a larger extent. Finally, in the limit of $r_{c} \rightarrow \infty$ these quantities for all $l$ states correspond to unity, as expected.

Table III and S2 in SM now report our estimated values of $T_{\mathbf{r}}^{\alpha}, T_{\mathbf{p}}^{\beta}$ and $T_{t}^{(\alpha, \beta)}$ for $2 p, 3 d$ and $4 f, 5 \mathrm{~g}$ states of CHA successively at the same $r_{c}$ 's of Table I and S1. These are carefully selected so as to cover small, moderate and large cavity radius. Once again, there exists no reference work for comparison. In all these four states, starting from some (-)ve value, $T_{\mathbf{r}}^{\alpha}$ 's advance monotonically with $r_{c}$ and finally reach the respective FHA behavior after some larger finite $r_{c}$. Like $R_{\mathbf{r}}^{\alpha}$ of Table I and S1, $T_{\mathbf{r}}^{\alpha}$ is also negative up to $r_{c} \leq 0.5$. 
TABLE IV: Tsallis entropies $T_{\mathbf{r}}^{\alpha}$ and $T_{\mathbf{p}}^{\beta}$ for all $l$ states corresponding to $n=10$, of CHA at various $r_{c}$, for $\alpha$ and $\beta$ as $\frac{3}{5}$ and 3 respectively. More details can be found in the text.

\begin{tabular}{|c|c|c|c|c|c|c|c|}
\hline 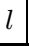 & $r_{c}=0.1$ & $r_{c}=0.5$ & $r_{c}=1$ & $r_{c}=10$ & $r_{c}=40$ & $r_{c}=80$ & $r_{c}=100$ \\
\hline \multicolumn{8}{|c|}{$T_{\mathbf{r}}^{\alpha}$} \\
\hline 0 & -2.28028155100 & -0.9836825500 & 0.9852090051 & 53.2532949166 & 299.6588765682 & 708.652348746 & 937.0301091469 \\
\hline 1 & -2.30589991419 & -1.1607438353 & 0.5774679920 & 46.4962802663 & 261.4396566474 & 620.0665476461 & 820.75069711 \\
\hline 2 & -2.30585532605 & -1.1605549274 & 0.5775561424 & 46.3871370347 & 258.8795701728 & 612.5075565192 & 811.1316848206 \\
\hline 3 & -2.30413187694 & -1.1487353757 & 0.6045090554 & 46.7495501415 & 259.3170313452 & 610.2456275455 & 807.5251254619 \\
\hline 4 & -2.30172366881 & -1.1321655705 & 0.6424491328 & 47.3096638083 & 261.2217971179 & 611.0280909941 & 807.072341736 \\
\hline 5 & -2.29880409954 & -1.1120499540 & 0.6885886895 & 48.0162247111 & 264.2344575738 & 614.6706053311 & 809.8947988758 \\
\hline 6 & -2.29545538209 & -1.0889560609 & 0.7416206960 & 48.8472617617 & 268.2127601388 & 621.1627157435 & 816.3075412867 \\
\hline 7 & -2.29184130513 & -1.0640097551 & 0.7989716149 & 49.7653312815 & 273.0158081039 & 630.500042316 & 826.5579416463 \\
\hline 8 & -2.28852393521 & -1.0410753919 & 0.8518014821 & 50.6415282666 & 278.1809736722 & 642.3612266162 & 840.5648183275 \\
\hline 9 & -2.28813664043 & -1.0382788056 & 35853232 & 50.8529116901 & 281.1761223672 & 653.6562832778 & 854.8383313138 \\
\hline \multicolumn{8}{|c|}{$T_{\mathbf{p}}^{\beta}$} \\
\hline 0 & 0.4999999999999998 & 0.49999999999 & 0.4999999998 & 0.49989313660 & -0.116508 & -39.789255 & -207.416554 \\
\hline 1 & 0.4999999999999991 & 0.49999999998 & 0.4999999991 & 0.49934534041 & -1.309576 & -103.015651 & -469.762391 \\
\hline 2 & 0.4999999999999981 & 0.49999999997 & 0.4999999981 & 0.49844386357 & -1.740324 & -170.679518 & -776.396253 \\
\hline 3 & 0.4999999999999966 & 0.49999999994 & 0.4999999966 & 0.49705220303 & -3.508707 & -313.589136 & -1045.065137 \\
\hline 4 & 0.4999999999999944 & 0.49 & 9944 & 9902295 & -8.404357 & -435.549958 & -1661.925267 \\
\hline 5 & 0.4999999999999908 & 0.49999999985 & 0.4999999908 & 0.49150326392 & -18.556282 & -534.047620 & -2456.963057 \\
\hline 6 & 0.4999999999999851 & 0.49999999976 & 0.4999999851 & 0.48597521532 & -37.620905 & -878.267905 & -3206.037005 \\
\hline 7 & 0.4999999999999754 & 0.49999999961 & 0.4999999755 & 0.47654334855 & -72.949846 & -2123.956362 & -5417.179246 \\
\hline 8 & 0.4999999999999579 & 0.49999999934 & 0.4999999580 & 0.45922740769 & -141.630950 & -5716.873607 & -15065.997051 \\
\hline 9 & 0.4999999999999197 & 0.49999999874 & 0.4999999198 & 0.42089205609 & -303.065930 & -16321.820720 & -53253.000550 \\
\hline
\end{tabular}

On the contrary, similar to $R_{\mathbf{p}}^{\beta}$ of Table I and S1, as $r_{c}$ progresses, $T_{\mathbf{p}}^{\beta}$ 's gradually decline from an initial result of $\approx \frac{1}{2}$, to large negative values (passing through a zero) at the end to merge with FHA result. Consequently, $T^{(\alpha, \beta)}$ 's in these circular states grow (starting from a small negative) with advancement of $r_{c}$ and then attain a positive maximum and finally fall off to particular FHA value (large negative). As observed for $R, T$ 's in both $r$, $p$ spaces follow the same order, viz., $T_{\mathbf{r}}^{\alpha}(5 g)>T_{\mathbf{r}}^{\alpha}(4 f)>T_{\mathbf{r}}^{\alpha}(3 d)>T_{\mathbf{r}}^{\alpha}(2 p)$ and $T_{\mathbf{p}}^{\beta}(5 g)>$ $T_{\mathbf{p}}^{\beta}(4 f)>T_{\mathbf{p}}^{\beta}(3 d)>T_{\mathbf{p}}^{\beta}(2 p)$ respectively. As usual, at $r_{c} \rightarrow \infty$ the order in $p$ space reverses to $T_{\mathbf{p}}^{\beta}(5 g)<T_{\mathbf{p}}^{\beta}(4 f)<T_{\mathbf{p}}^{\beta}(3 d)<T_{\mathbf{p}}^{\beta}(2 p)$. Then we proceed for $T_{\mathbf{r}}^{\alpha}, T_{\mathbf{p}}^{\beta}$ for $n=10$ states at same $r_{c}$ of Table II. Similar to $R_{\mathbf{r}}^{\alpha}, T_{\mathbf{r}}^{\alpha}$ 's remain $(-)$ ve for all $l$ at $r_{c}<1$ and (+)ve for $r_{c}>1$. For each $l$, they increase with $r_{c}$. Again analogous to $R_{\mathbf{r}}^{\alpha}$, at each $r_{c}, T_{\mathbf{r}}^{\alpha}$ first falls down to a minimum and finally grow with $l$. For first three $r_{c}$, these minima occur at $l=1$. For $r_{c}=10,40$ these minima are found at $l=2$, whereas for $r_{c}=40$ and 100 these minima 
TABLE V: $S_{\mathbf{r}}, S_{\mathbf{p}}$ and $S_{t}$ for $2 p, 3 d$ states of CHA at various $r_{c}$. Reference values [10] for $2 p$ and $3 d$ states quoted here include angular contributions $S_{(\theta, \phi)}$, along with $S_{r}$ results given in the Supplementary Tables VIII $(2 p)$ and XIV $(3 d)$ respectively. See text for details.

\begin{tabular}{|c|c|c|c|c|c|c|c|}
\hline$r_{c}$ & $S_{\mathbf{r}}$ & $S_{\mathbf{p}}$ & $S_{t}$ & $r_{c}$ & $S_{\mathbf{r}}$ & $S_{\mathbf{p}}$ & $S_{t}$ \\
\hline \multicolumn{4}{|c|}{$2 p^{\ddagger ! !}$} & \multicolumn{4}{|c|}{$3 d^{\ddagger}, \boldsymbol{\Upsilon}$} \\
\hline 0.1 & -6.3897304044 & 13.4182 & 7.0285 & 0.1 & -6.3559834637 & 14.0037 & 7.6477 \\
\hline $0.2^{\S}$ & -4.3126791264 & 11.3396 & 7.0270 & $0.2^{\dagger}$ & -4.2772270811 & 11.9244 & 7.6472 \\
\hline 0.3 & -3.0987163770 & 10.1240 & 7.0253 & 0.3 & -3.0615253452 & 10.7082 & 7.6467 \\
\hline 0.5 & -1.5712349891 & 8.5934 & 7.0222 & 0.5 & -1.5304613573 & 9.1761 & 7.6457 \\
\hline 0.8 & -0.1690563768 & 7.1867 & 7.0177 & 0.8 & -0.1226356677 & 7.7668 & 7.6442 \\
\hline $1^{\S}$ & 0.4949160183 & 6.5198 & 7.0147 & $1^{\dagger}$ & 0.5452929876 & 7.0980 & 7.6432 \\
\hline $5^{\S}$ & 5.1596858896 & 1.83592 & 6.9956 & 7.5 & 6.5142648552 & 1.124506 & 7.63877 \\
\hline 15 & 7.2120252839 & 0.060324 & 7.272349 & 15 & 8.3843979910 & -0.61531257 & 7.76908542 \\
\hline 25 & 7.2648183351 & 0.0423328 & 7.3071511 & $50^{\dagger}$ & 9.3456307493 & -1.232729196 & 8.1129015533 \\
\hline 45 & 7.2648971157 & 0.04242079 & 7.3073179 & $100^{\dagger}$ & 9.3456341991 & -1.232717246 & 8.112916953 \\
\hline
\end{tabular}

${ }^{\S}$ Literature results [10] of $\left(S_{\mathbf{r}}, S_{\mathbf{p}}, S_{t}\right)$ at $r_{c}=0.2,1,5$ in $2 p$ state are: $(-4.3126791236,11.3396511782,7.0269720545)$, $(0.4949160211,6.5198502152,7.0147662362)$ and $(5.1596858926,1.8359293466,6.9956152391)$ respectively.

${ }^{\dagger}$ Literature results [10] of $\left(S_{\mathbf{r}}, S_{\mathbf{p}}, S_{t}\right)$ at $r_{c}=0.2,1,50,100$ in $3 d$ state are: $(-4.2772270783,11.9244780687,6.8797080015)$, (0.5452929905, 7.098078228, 7.6433712186), (9.3456307877, -1.2327291038, 8.112901684), and $(9.345634202,-1.2327172441,8.112916958)$ respectively.

¥Reference values are added with respective $S_{(\theta, \phi)}$ values $(2.0990786249678,2.0411250061339)$ of $2 p$ and $3 d$ states.

${ }^{!} S_{\mathbf{r}}, S_{\mathbf{p}}, S_{t}$ in FHA for $2 p$ states $(|m|=0)$ are: $7.264897118452,0.042420799485,7.307317917937$.

${ }^{\top} S_{\mathbf{r}}, S_{\mathbf{p}}, S_{t}$ in FHA for $3 d$ states $(|m|=0)$ are: 9.345634202074, $-1.232717244109,8.112916957965$.

appear at $l=3$ and 4 successively. On the other hand, $T_{\mathbf{p}}^{\beta}$ decrease with progress of $r_{c}$. Further, in all seven $r_{c}, T_{\mathbf{p}}^{\beta}$ 's consistently decay with $l$, although the extent is very less till $r_{c}=1$ and assumes significance only after $r_{c}>5$ or so. As there is a good resemblance of the behavior of CHA and FHA ratios in Rényi and Tsallis entropies in $r$ and $p$ spaces, one can expect and predict the qualitative nature of similar plots for $T$ for these states, and hence omitted here.

Let us now shift our focus on Table $\mathrm{V}$ and $\mathrm{S} 3$ in SM, where $S_{\mathbf{r}}, S_{\mathbf{p}}$ and $S_{t}$ of CHA are probed for four low-lying circular states corresponding to $l=1-4$. The same set of $r_{c}$ of Table I and $\mathrm{S} 1$ is adopted. This time, a handful of results are available in the literature for $2 p$ (at $\left.r_{c}=0.2,1,5\right)$ and $3 d\left(r_{c}=0.2,1,50,100\right)$ states of CHA, which are duly quoted. Present results show good agreement with reference data in all occasions. $S_{\mathbf{r}}, S_{\mathbf{p}}, S_{t}$ portray analogous behavior to those of $R_{\mathbf{r}}^{\alpha}, R_{\mathbf{p}}^{\beta}$ and $R^{(\alpha, \beta)}$ respectively. Similar to $R_{\mathbf{r}}^{\alpha}, S_{\mathbf{r}}$ also take $(-)$ ve values for all four states at $r_{c}<1$ region and evolve continuously 
TABLE VI: $S_{\mathbf{r}}$ and $S_{\mathbf{p}}$ for all $l$ states corresponding to $n=10$, of CHA at representative $r_{c}$ values, in top and bottom segments. For more details, consult text.

\begin{tabular}{|c|c|c|c|c|c|c|c|}
\hline$l$ & $r_{c}=0.1$ & $r_{c}=0.5$ & $r_{c}=1$ & $r_{c}=10$ & $r_{c}=40$ & $r_{c}=80$ & $r_{c}=100$ \\
\hline \multicolumn{8}{|c|}{$S_{\mathbf{r}}$} \\
\hline 0 & -6.6336010412 & -1.8032959712 & 0.2787117983 & 7.2442147026 & 11.5877476335 & 13.8154049673 & 14.5452382494 \\
\hline 1 & -6.9827167662 & -2.1537824035 & -0.0735403924 & 6.8533262967 & 11.1225960687 & 13.3570558294 & 14.0927363946 \\
\hline 2 & -6.9592958229 & -2.1308141271 & -0.0511509821 & 6.8629221900 & 11.0765559826 & 13.2799996428 & 14.0148698959 \\
\hline 3 & -6.8974004176 & -2.0691277981 & 0.0102684892 & 6.9184633212 & 11.0999960757 & 13.2630164065 & 13.9887469229 \\
\hline 4 & -6.8231110824 & -1.9949407785 & 0.0843246799 & 6.9895792871 & 11.1527172455 & 13.2805941590 & 13.9928101925 \\
\hline 5 & -6.7421222230 & -1.9139917010 & 0.1652222750 & 7.0691946490 & 11.2222138846 & 13.3235593289 & 14.0216304087 \\
\hline 6 & -6.6566958653 & -1.8285574196 & 0.2506653439 & 7.1545675577 & 11.3032904456 & 13.3866129318 & 14.0720358679 \\
\hline 7 & -6.5693810669 & -1.7411860014 & 0.3381068528 & 7.2431276172 & 11.3927769257 & 13.4664617170 & 14.1417536426 \\
\hline 8 & -6.4873245243 & -1.6590042222 & 0.4204449123 & 7.3282175171 & 11.4854342953 & 13.5598715121 & 14.2284568921 \\
\hline 9 & -6.4437380685 & -1.6151489690 & 0.4646366944 & 7.3785621302 & 11.5570097332 & 13.6533937166 & 14.3196849974 \\
\hline \multicolumn{8}{|c|}{$S_{\mathbf{p}}$} \\
\hline 0 & 18.225 & 13.399 & 11.324 & 4.710 & 0.711 & -1.206 & -1.8374 \\
\hline 1 & 17.685 & 12.858 & 10.7811 & 4.0149 & 0.4927 & -1.53 & -2.29 \\
\hline 2 & 17.512 & 12.684 & 10.6067 & 3.780 & 0.308 & -1.70 & -2.289 \\
\hline 3 & 17.367 & 12.539 & 10.461 & 3.605 & -0.032 & -1.78 & -2.412 \\
\hline 4 & 17.219 & 12.3919 & 10.313 & 3.440 & -0.28 & -1.872 & -2.557 \\
\hline 5 & 17.063 & 12.2355 & 10.156 & 3.270 & -0.581 & -2.09 & -2.6719 \\
\hline 6 & 16.889 & 12.061 & 11.982 & 3.087 & -0.875 & -2.427 & -2.906 \\
\hline 7 & 16.689 & 12.861 & 11.782 & 2.880 & -1.171 & -2.84 & -3.284 \\
\hline 8 & 16.447 & 12.618 & 11.539 & 2.632 & -1.482 & -3.31 & -3.792 \\
\hline 9 & 16.105 & 12.276 & 11.197 & 2.285 & -1.871 & -3.871 & -4.4326 \\
\hline
\end{tabular}
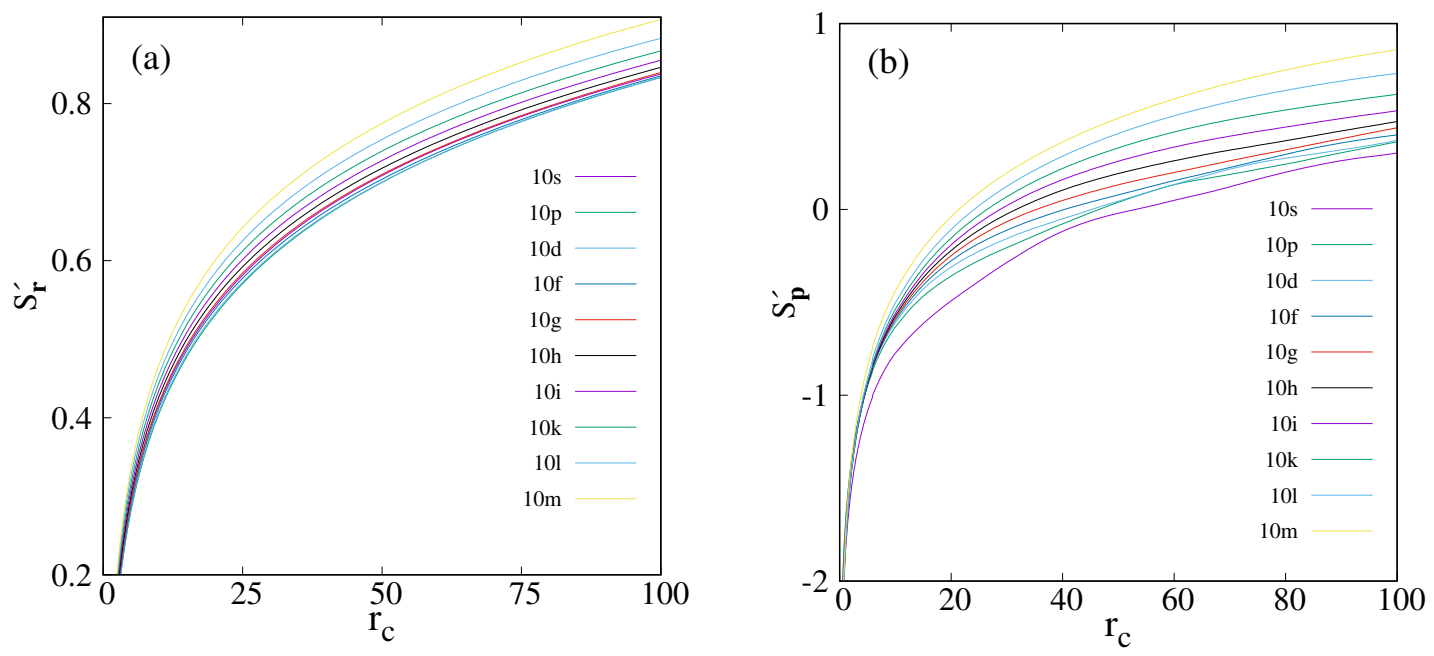

FIG. 2: Variation of $S_{\mathbf{r}}^{\prime}, S_{\mathbf{p}}^{\prime}$ of CHA with $r_{c}$, for $n=10$ states, in panels (a), (b). See text for detail. 
with growth of $r_{c}$ before reaching the FHA-limit at large $r_{c}$. On the contrary $S_{\mathbf{p}}$, like $R_{\mathbf{p}}^{\beta}$, shows a reverse nature of $S_{\mathbf{r}}$. $S_{\mathbf{p}}$ for $l=1-4(n=l+1)$ states, starting from $(+)$ ve numerical values, decline as $r_{c}$ develops before merging with FHA limit. Consequently, $S_{t}$, falls to reach a minimum and then elevates to attain FHA value. Furthermore, $S_{\mathbf{r}}$ imprints different pattern to $R_{\mathbf{r}}^{\alpha}$ but $S_{\mathbf{p}}$ delineates similar leaning to $R_{\mathbf{p}}^{\beta}$ in smaller $r_{c}$. The observed trend in $r, p$ spaces is slightly unusual: $S_{\mathbf{r}}(4 f)>S_{\mathbf{r}}(5 g)>S_{\mathbf{r}}(3 d)>S_{\mathbf{r}}(2 p)$ and $S_{\mathbf{p}}(5 g)>S_{\mathbf{p}}(4 f)>S_{\mathbf{p}}(3 d)>S_{\mathbf{p}}(2 p)$ respectively. As usual at $r_{c} \rightarrow \infty$ the order in $r, p$ space reorganize to $S_{\mathbf{r}}(5 g)>S_{\mathbf{r}}(4 f)>S_{\mathbf{r}}(3 d)>S_{\mathbf{r}}(2 p)$ and $S_{\mathbf{p}}(5 g)<S_{\mathbf{p}}(4 f)<S_{\mathbf{p}}(3 d)<S_{\mathbf{p}}(2 p)$. As a next step, Table VI supplies $S_{\mathbf{r}}, S_{\mathbf{p}}$ for ten $l$ states corresponding to $n=10$ at same selected $r_{c}$ set discussed before. $S_{\mathbf{r}}$ remains $(-)$ ve for all $l$ at first two $r_{c}$ 's. Interestingly, at $r_{c}=1, S_{\mathbf{r}}$ is $(-)$ ve only for $l=1,2$. In rest of the situation, $S_{\mathbf{r}}$ takes on $(+)$ ve values. $S_{\mathbf{r}}$, in all $r_{c}$ 's at first diminish with $l$, attain some minima and then gains. $S_{\mathrm{r}}$ reaches minimum at $l=1$ for first four $r_{c}$ values. At $r_{c}=40$ this mimimum shifts to $l=2$, whereas for $r_{c}=80$ and 100 these minima arive at $l=3$. But in $p$ space, $S_{\mathbf{p}}$ imitates $R_{\mathbf{p}}^{\beta}$. Hence, like $R_{\mathbf{p}}^{\beta}$, here $S_{\mathbf{p}}$ collapses with rise of $l$. Here again the nature of $S_{\mathbf{r}}, S_{\mathbf{p}}$ variation for $10 l$ states remains akin to respective $R_{\mathbf{r}}^{\alpha}, R_{\mathbf{p}}^{\beta}$ changes. Like other states reported here, $S_{\mathbf{r}}$ progresses and $S_{\mathbf{p}}$ reduces with growth of $r_{c}$.

Now we move on to Fig. 2 where following the strategy of Fig. 1, the relevant pair of unit-less ratios viz., $S_{\mathbf{r}}^{\prime}=\left(\frac{S_{\mathbf{r}}(C H A)}{S_{\mathbf{r}}(F H A)}\right)$ and $S_{\mathbf{p}}^{\prime}=\left(\frac{S_{\mathbf{p}}(C H A)}{S_{\mathbf{p}}(F H A)}\right)$ are displayed for all $l$ states having $n=10$. Drawing reference to Table VI, one notices that, for any given $n, l$ quantum number, $S_{\mathbf{r}}$ rises and $S_{\mathbf{p}}$ falls continuously as $r_{c}$ proceeds to reach their corresponding maximum and minimum limits at $r_{c} \rightarrow \infty$. Further, this limiting value in $S_{\mathbf{p}}$ relates to (-) ve sign (not obvious from Table VI; but further extension of $r_{c}$ assures that). Thus both these ratios are bounded to their maximum values to unity. Hence, they both exhibit similar trend in behavior, i.e., grow up with $r_{c}$ signifying delocalization of the system. In the entire range of $r_{c}$ the values of both $S_{\mathbf{r}}^{\prime}$ and $S_{\mathbf{p}}^{\prime}$ abate with $l$, which suggests that, states with higher number of nodes, experience confinement to a greater extent.

Now Fig. 3 inscribes variation of $R_{\mathbf{r}}^{\alpha}, S_{\mathbf{r}}\left(I E_{\mathbf{r}}\right)$ and $R_{\mathbf{p}}^{\beta}, S_{\mathbf{p}}\left(I E_{\mathbf{p}}\right)$ of $n=10$ states with change of $l$ in left and right columns labeled A, B. Here $I E$ stands for Information Entropy and in what follows this is loosely used to signify any or all of the measures discussed in this communication. These are given at five representative $r_{c}$ 's namely, $0.1,10,60,100, \infty$, identified by (a)-(e) in parentheses. At first four finite $r_{c}$ 's, both $R_{\mathbf{r}}^{\alpha}$ and $S_{\mathbf{r}}$ fall off to 

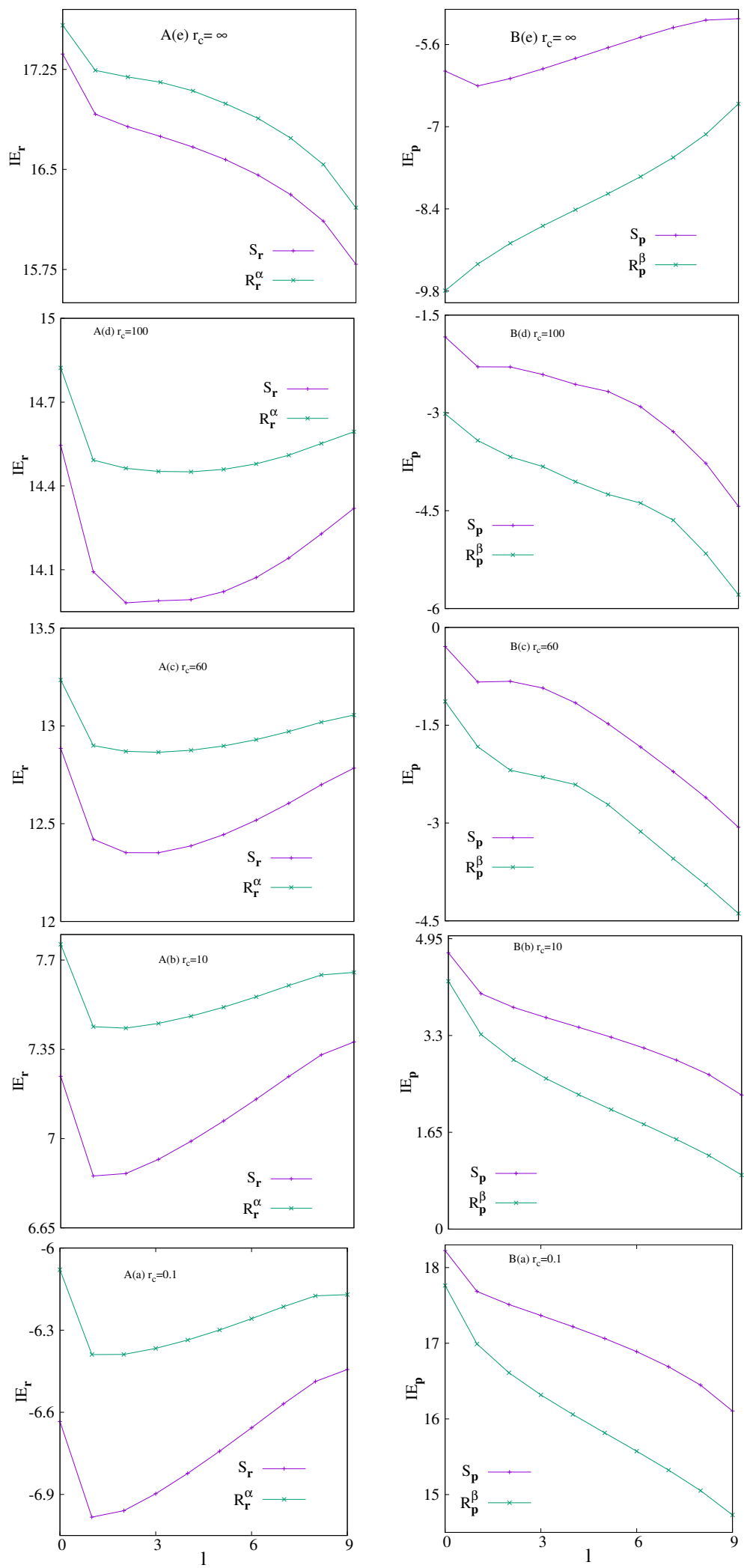

FIG. 3: Variation of $R_{\mathbf{r}}^{\alpha}, S_{\mathbf{r}}\left(I E_{\mathbf{r}}\right)$ and $R_{\mathbf{p}}^{\beta}, S_{\mathbf{p}}\left(I E_{\mathbf{p}}\right)$ against $l$ for $n=10$ states of CHA in A and B columns. Five specific $r_{c}$ 's $(0.1,10,60,100, \infty)$ are identified in parentheses (a), (b), (c), (d), (e) respectively. See text for detail. 
TABLE VII: $E_{\mathbf{r}}, E_{\mathbf{p}}, E_{t}$ for $2 p, 3 d$ states of CHA at various $r_{c}$. For more details, see text.

\begin{tabular}{llll|llll}
\hline \hline$r_{c}$ & $E_{\mathbf{r}}$ & $E_{\mathbf{p}}$ & $E_{t}$ & $r_{c}$ & $E_{\mathbf{r}}$ & $E_{\mathbf{p}}$ & $E_{t}$ \\
\hline & & $2 p^{!}$ & & & & $3 d^{\boldsymbol{\Psi}}$ & \\
\hline 0.1 & 805.746259389130 & 0.00000227289 & 0.0018313726 & 0.1 & 851.986890492579 & 0.00000137674 & 0.0011729644 \\
0.2 & 101.040180954047 & 0.00001814520 & 0.0018333943 & 0.2 & 106.590831757296 & 0.00001100401 & 0.0011729266 \\
0.3 & 30.0353202004482 & 0.00006110958 & 0.0018354458 & 0.3 & 31.610240295272 & 0.00003710446 & 0.0011728809 \\
0.5 & 6.5311618804191 & 0.00028167391 & 0.0018396579 & 0.5 & 6.840055708558 & 0.00017145905 & 0.0011727895 \\
0.8 & 1.6113769393302 & 0.00114576730 & 0.001846263 & 0.8 & 1.674574222639 & 0.00070026869 & 0.0011726519 \\
1 & 0.8311099104230 & 0.00222698556 & 0.0018508698 & 1 & 0.859020100126 & 0.00136499761 & 0.0011725604 \\
5 & 0.0083268168966 & 0.23942780273 & 0.0019936715 & 7.5 & 0.002245898234 & 0.52169438759 & 0.0011716725 \\
15 & 0.0014334774547 & 1.90861683331 & 0.0027359592 & 15 & 0.000370433690 & 3.22168315792 & 0.00119342 \\
25 & 0.0013988503823 & 1.97640112599 & 0.0027646895 & 50 & 0.00001394232 & 35.52898842628 & 0.0004953565 \\
45 & 0.0013988227375 & 1.97576308974 & 0.0027637423 & 100 & 0.000172694164 & 7.10053419923 & 0.0012262208 \\
\hline \hline
\end{tabular}

${ }^{!} E_{\mathbf{r}}, E_{\mathbf{p}}, E_{t}$ in FHA for $2 p$ states $(|m|=0)$ are: $0.001398822737,1.975763081024,0.002763742330$.

${ }^{\top} E_{\mathbf{r}}, E_{\mathbf{p}}, E_{t}$ in FHA for $3 d$ states $(|m|=0)$ are: $0.000172694164,7.1005342704468,0.0012262208417$.

reach certain minima and then improve with $l$ in panels $\mathrm{A}(\mathrm{a})-\mathrm{A}(\mathrm{d})$. Positions of both these minima shift to right as $r_{c}$ is raised. Locations of these lowest points correspond to $l$ values in accordance with Tables II and IV discussed before. However, in $r_{c}=\infty$ limit, for both measures in $r$ space, the minima disappear; rather there is a steady decline with $l$ which passes through a plateau region. The respective $p$-space quantities in first four panels $\mathrm{B}(\mathrm{a})-\mathrm{B}(\mathrm{d})$ on the right side, decline gradually with $l$. However, in top fifth panel $\mathrm{B}(\mathrm{e})$, both of them are raised with increment of $l$. This study simply reveals distinctly separate behavior of a $\mathrm{H}$ atom from free to confined environment. In case of FHA, both spread and radial nodes of an wave-function reduce with $l$; hence $R_{\mathbf{r}}^{\alpha}$ as well as $S_{\mathbf{r}}$ diminish while $R_{\mathbf{p}}^{\beta}$ increases with uprise of $l$. Further, there appears a shallow minimum in $S_{p}$ for FHA. But in the confinement scenario, there exists an interplay between two mutually opposing factors: (i) radial confinement (favoring localization) and (ii) accumulation of radial nodes with reduction in $l$ (promoting delocalization). Hence, such minima appear in IE plots in panels $A(a)-A(d)$ and $B(a)-B(d)$.

Next we move on to discuss the last measure in this work, namely, $E$ for same four nonzero $l$ states as considered for $R, T, S$, in Table VII and S4 in SM. Once again no literature results could be found to compare. Generally speaking, behavior of $E$ is usually reverse to those of $R, T, S$ in Tables I, III, V and S1-S3. Thus for all four states, $E_{\mathbf{r}}$ 's diminish, while $E_{\mathbf{p}}$ advance with surge of $r_{c}$. As $r_{c}$ approaches zero, $E_{\mathbf{r}}$ obeys the trend $E_{\mathbf{r}}(5 g)>E_{\mathbf{r}}(4 f)>$ 
TABLE VIII: $E_{\mathbf{r}}$ and $E_{\mathbf{p}}$ for all $l$ states corresponding to $n=10$, of CHA at representative $r_{c}$ values, in top and bottom sections. For more details, consult text.

\begin{tabular}{|c|c|c|c|c|c|c|c|}
\hline \multicolumn{8}{|c|}{$E_{\mathbf{r}}$} \\
\hline$l$ & $r_{c}=0.1$ & $r_{c}=0.5$ & $r_{c}=1$ & $r_{c}=10$ & $r_{c}=40$ & $r_{c}=80$ & $r_{c}=100$ \\
\hline 0 & 7747.631350106 & 62.389881644 & 7.861047634 & 0.008550277 & 0.0000975413 & 0.0000072401 & 0.0000029779 \\
\hline 1 & 6063.245277416 & 48.6353842949 & 6.0994482170 & 0.00641025319 & 0.0000996112 & 0.0000094350 & 0.0000040864 \\
\hline 2 & 4522.362533153 & 36.2403977184 & 4.5396157540 & 0.00470099482 & 0.0000773147 & 0.0000085650 & 0.0000039070 \\
\hline 3 & 3530.138368622 & 28.2761820347 & 3.5399931260 & 0.00363570042 & 0.0000603524 & 0.0000072797 & 0.0000034687 \\
\hline 4 & 2838.936113913 & 22.7331219908 & 2.8450195749 & 0.00290537538 & 0.0000480878 & 0.0000060612 & 0.0000029830 \\
\hline 5 & 2326.540075860 & 18.6259402411 & 2.3303725215 & 0.00236895765 & 0.0000389288 & 0.0000050118 & 0.0000025222 \\
\hline 6 & 1930.662444626 & 15.4535730094 & 1.9329918593 & 0.00195674165 & 0.0000318467 & 0.0000041310 & 0.0000021092 \\
\hline 7 & 1618.128828159 & 12.9493540013 & 1.6193470206 & 0.00163196755 & 0.0000262324 & 0.0000033940 & 0.0000017474 \\
\hline 8 & 1375.046723046 & 11.0013562943 & 1.3753245996 & 0.00137841885 & 0.0000217702 & 0.0000027783 & 0.0000014345 \\
\hline 9 & 1224.322475119 & 9.79175302714 & 1.2235277207 & 0.00121564677 & 0.0000186122 & 0.0000022894 & 0.0000011795 \\
\hline \multicolumn{8}{|c|}{$E_{\mathbf{p}}$} \\
\hline 0 & 0.00000001732663 & 0.000002159204 & 0.000017186150 & 0.01275050 & 0.918902 & 6.52106 & 12.55735 \\
\hline 1 & 0.00000003453069 & 0.000004307362 & 0.00003435147 & 0.02945819 & 1.366699 & 10.40162 & 22.83099 \\
\hline 2 & 0.00000004594109 & 0.000005733162 & 0.00004575769 & 0.04151677 & 1.479949 & 13.08729 & 25.89834 \\
\hline 3 & 0.0000000570426 & 0.00000712045 & 0.00005685540 & 0.05313830 & 1.937268 & 15.702434 & 29.25821 \\
\hline 4 & 0.0000000693689 & 0.000008660817 & 0.00006917634 & 0.06584220 & 2.714584 & 16.70624 & 34.56930 \\
\hline 5 & 0.0000000840379 & 0.00001049398 & 0.00008383898 & 0.0807910 & 3.767910 & 18.52554 & 38.28028 \\
\hline 6 & 0.0000001024152 & 0.00001279075 & 0.0001022107 & 0.0993984 & 5.123417 & 24.45627 & 43.23403 \\
\hline 7 & 0.0000001266503 & 0.00001582009 & 0.0001264462 & 0.12388312 & 6.89702 & 37.097591 & 58.60068 \\
\hline 8 & 0.0000001609259 & 0.00002010565 & 0.0001607428 & 0.1585969 & 9.37541 & 59.3034 & 96.4456 \\
\hline 9 & 0.00000021939962 & 0.00002741951 & 0.00021930107 & 0.2181656 & 3.56075 & 99.301658 & 178.3531 \\
\hline
\end{tabular}

$E_{\mathbf{r}}(3 d)>E_{\mathbf{r}}(2 p)$ which gets reversed to $E_{\mathbf{r}}(2 p)>E_{\mathbf{r}}(3 d)>E_{\mathbf{r}}(4 f)>E_{\mathbf{r}}(5 g)$ in opposite $r_{c}$ limit. Contrariwise, $E_{\mathbf{p}}$ shows exactly opposite trend from its $r$-space counterpart at both small and large $r_{c}$ regions. Finally, Table VIII features $E_{\mathbf{r}}, E_{\mathbf{p}}$ for $n=10$ states in upper, lower portions. For all ten $l, E_{\mathbf{r}}$ 's collapse with accrual of $r_{c}$. At first four $r_{c}$ values $E_{\mathbf{r}}$ droops down with rise of $l$. But, in other three $r_{c}$ values there appear maxima at $l=1$. In $p$ space, these patterns are completely reverse from $r$-space counterparts. Trends in $E_{\mathbf{r}}, E_{\mathbf{p}}$ with respect to $r_{c}$ complement the findings of $R, T, S$.

Figure 4 now sketches the logarithmic change of $E_{\mathbf{r}}, E_{\mathbf{p}}$ with $r_{c}$ for $n=10$ states in panels (a), (b). In contrast to $R$ and $S, E_{\mathbf{r}}$ lessens whereas $E_{\mathbf{p}}$ strengthens as $r_{c}$ grows, for any given $n, l$. Like all other measures, they both eventually merge to their FHA values. Convergence of $E_{\mathbf{p}}$ is not obvious from panel (b); however this can be verified upon extending $r_{c}$ to some sufficiently large value. As in case of Tables VII and S4, this result again consolidates our 

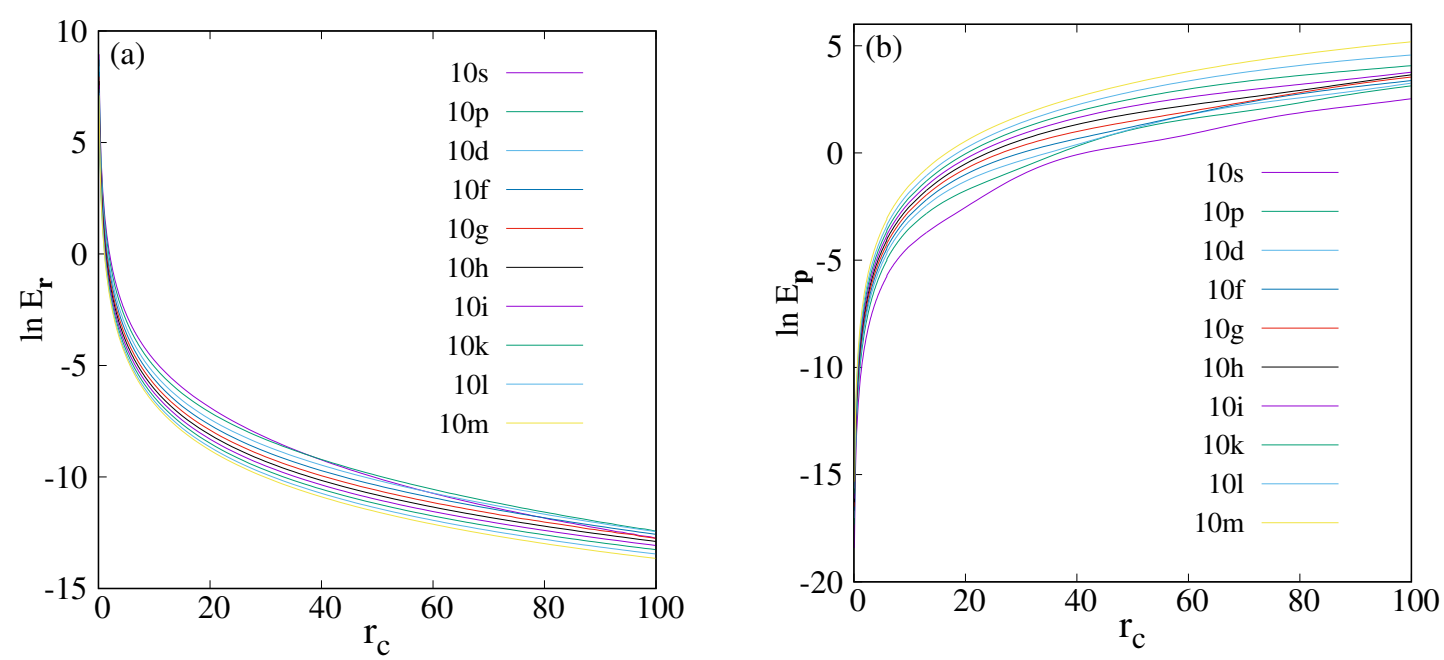

FIG. 4: Logarithmic changes of $E_{\mathbf{r}}, E_{\mathbf{p}}$ of CHA with $r_{c}$, for $n=10$, in panels (a), (b). Consult text for detail.

previous discussion on $R, T, S$ that, relaxation in confinement facilitates delocalization.

Lastly in order to understand these measures with charge, Fig. 5 displays behavioral patterns of $R, S, E$ in conjugate $r, p$ spaces. In this occasion, we limit the discussion to ground state only, as it can be trivially extended for other states. These are followed at seven particular $Z$, viz., 1, 2, 3, 4, 5, 10, 15. Bottom and top rows characterizing $r$ - $p$ space properties are denoted by A, B, while $R, S, E$ are identified by parentheses (a), (b), (c) respectively. Note that $T$ behavior is similar in fashion to $R$; thus lead to common interpretation and hence not reported in this figure. It is seen from bottom three panels that, $R_{\mathrm{r}}^{\alpha}, S_{\mathrm{r}}$ advance, whereas, $E_{\mathrm{r}}$ falls off with an escalation in $Z$. Whereas from top row it is observed that $R_{\mathbf{p}}^{\beta}, S_{\mathbf{p}}$ decay while $E_{\mathbf{p}}$ intensifies with increment of $Z$. In $\mathrm{B}(\mathrm{c})$ segment, $Z=1, Z=2$ graphs also show similar behavior like other $Z$ values (not clear from panel $\mathrm{B}(\mathrm{c}))$. A careful scrutiny of these measures with respect to $Z$ suggests that, for any arbitrary $n l$ state, an increase in $Z$ promotes localization. Hence, the electron density gets tightened as one goes to heavier atoms. This strengthens the trend in results of various observed chemical phenomena like electronegativity, ionisation potential, hard-soft interaction etc., in atomic systems. Table portraying the values of $R, T, S, E$ at various $Z$ are not given here. Because, such tables for $R, T, S, E$ can easily be constructed with the help of their definition in section II and data provided in section III. 

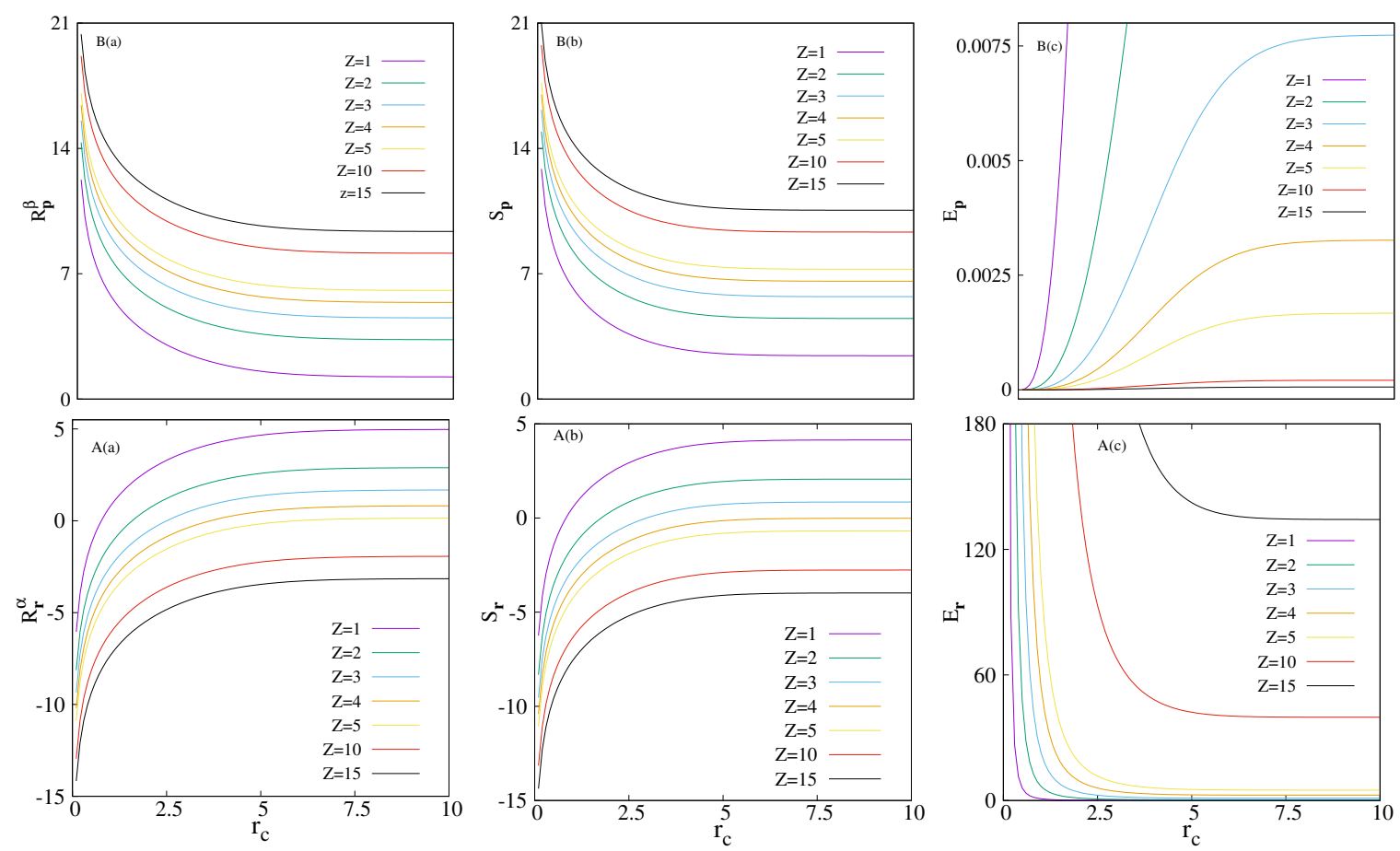

FIG. 5: Nature of $R_{\mathbf{r}}^{\alpha}, S_{\mathbf{r}}, E_{\mathbf{r}}$ (A) and $R_{\mathbf{p}}^{\beta}, S_{\mathbf{p}}, E_{\mathbf{p}}$ (B) in $r$ (lower) and $p$ (upper) spaces, for seven selected $Z$. R, S, E are labeled by parentheses (a), (b), (c) respectively. Ground-state results are given for $\alpha=\frac{3}{5}, \beta=3$. See text for details.

\section{FUTURE AND OUTLOOK}

Information-theoretic measures like $R, T, S, E$ are explored for $l \neq 0$ states of CHA in both $r, p$ spaces. Accurate results for combined measures (radial plus angular) are provided for $2 p, 3 d, 4 f, 5 g$ and $n=10$ states of CHA, keeping $m$ fixed at zero. Except for very recent publication of $S_{\mathbf{r}}, S_{\mathbf{p}}$ in $2 p, 3 d$ states, all these quantities are reported for first time. It is found that at small $r_{c}$, with growth of $n, R_{\mathbf{r}}^{\alpha}, T_{\mathbf{r}}^{\alpha}$ build up while $E_{\mathbf{r}}$ deteriorate. Beside this, $R_{\mathbf{r}}^{\alpha}, T_{\mathbf{r}}^{\alpha}, S_{\mathbf{r}}$ pass through a minimum with addition of $l$. Further, an investigation on $n=10$ states has been made to get an idea about the high-lying states of CHA. It is realized that, they may be exploited to analyze the spread as well as diffused nature of Rydberg-hydrogenic states. Additionally, scaling property has been utilized to ascertain the effect of atomic number on IE of confined atomic systems. Actually, upgradation in $Z$ strengthens the $r$-space electron density of any arbitrary $n l$ state of CHA. An examination of these quantities in the realm of Rydberg states under various types of confined environment may be worthwhile to consider. A collateral inspection of many-electron atomic systems 
regarding IE and periodic properties would be highly desirable.

\section{ACKNOWLEDGEMENT}

Financial support from DST SERB, New Delhi, India (sanction order: EMR/2014/000838) is gratefully acknowledged. NM thanks DST SERB, New Delhi, India, for a National-post-doctoral fellowship (sanction order: PDF/2016/000014/CS).

[1] N. Mukherjee and A. K. Roy, Int. J. Quant. Chem. (accepted).

[2] S.-Y. Ch'en and M. Takeo, Rev. Mod. Phys. 29, 20 (1957).

[3] W. Jaskólski, Phys. Rep. 271, 1 (1996).

[4] J. R. Sabin, E. Brändas and S. A. Cruz (Eds.), The Theory of Confined Quantum Systems, Parts I and II, Advances in Quantum Chemistry, Vols. 57 and 58 (Academic Press, 2009).

[5] J. Katriel and H. E. Montgomery Jr., J. Chem. Phys. 137, 114109 (2012).

[6] K. D. Sen (Ed.), Electronic Structure of Quantum Confined Atoms and Molecules, (Springer, Switzerland, 2014).

[7] H. Pang, W-S. Dai and M. Xie, J. Phys. A 44, 365001 (2011).

[8] A. Michels, J. de Boer and A. Bijl, Physica 4, 981 (1937).

[9] N. Aquino, A. Flores-Riveros and J. F. Rivas-Silva, Phys. Lett. A 377, 2062 (2013).

[10] L. G. Jiao, L. R. Zan, Y. Z. Zhang and Y. K. Ho, Int. J. Quant. Chem. 117, e25375 (2017).

[11] N. Sobrino-Coll, D. Puertas-Centeno, I. V. Toranzo and J. S. Dehesa, J. Stat. Mech. 8, 083102 (2017).

[12] D. Puertas-Centeno, N. M. Temme, I. V. Toranzo and J. S. Dehesa, J. Math. Phys. 58, $103302(2017)$.

[13] S. Goldman and C. Joslin, J. Phys. Chem. 96, 6021 (1992).

[14] N. Aquino A., Int. J. Quant. Chem. 54, 107 (1995).

[15] J. Garza, R. Vargas and A. Vela, Phys. Rev. E 58, 3949 (1998).

[16] C. Laughlin, B. L. Burrows and M. Cohen, J. Phys. B 35, 701 (2002).

[17] B. L. Burrows and M. Cohen, Int. J. Quant. Chem. 106, 478 (2006).

[18] N. Aquino, G. Campoy and H. E. Montgomery Jr. , Int. J. Quant. Chem. 107, 1548 (2007). 
[19] D. Baye and K. D. Sen, Phys. Rev. E 78, 026701 (2008).

[20] H. Ciftci, R. L. Hall and N. Saad, Int. J. Quant. Chem. 109, 931 (2009).

[21] H. E. Montgomery and K. D. Sen, Phys. Lett. A 376, 1992 (2012).

[22] R. Cabrera-Trujillo and S. A. Cruz, Phys. Rev. A 87, 012502 (2013).

[23] A. K. Roy, Int. J. Quant. Chem. 115, 937 (2015); ibid., 116, 953 (2016).

[24] A. Solórzano, N. Aquino and A. Flores-Riveros, Can. J. Phys. 94, 894 (2016).

[25] G.-H. Sun, M. A. Aoki and S.-H. Dong, Chin. Phys. B 22, 050302 (2013).

[26] G.-H. Sun, S.-H. Dong and N. Saad, Ann. Phys. (Berlin) 525, 934 (2013).

[27] W. A. Yahya, K. J. Oyewumi and K. D. Sen, Int. J. Quant. Chem. 115, 1543 (2015).

[28] S. Dong, G.-H. Sun, S.-H. Dong and J. P. Draayer, Phys. Lett. A 378, 124 (2014).

[29] R. Valencia-Torres, G.-H. Sun and S.-H. Dong, Phys. Scr. 90, 035205 (2015).

[30] G.-H. Sun, P. Duan, C.-N. Oscar and S.-H. Dong, Chin. Phys. B 24, 100303 (2015).

[31] G. Yañez-Navarro, G.-H. Sun, T. Dytrych, K. D. Launey, S.-H. Dong and J. P. Draayer, Ann. Phys. 348, 153 (2014).

[32] X.-D. Song, G.-H. Sun and S.-H. Dong, Phys. Lett. A 379, 1402 (2015).

[33] G.-H. Sun, S.-H. Dong, K. D. Launey, T. Dytrych and J. P. Draayer, Int. J. Quant. Chem. 115, $891(2015)$.

[34] N. Mukherjee, A. Roy and A. K. Roy, Ann. Phys. (Berlin) 527, 825, (2015).

[35] N. Mukherjee and A. K. Roy, Ann. Phys. (Berlin) 528, 412, (2016).

[36] A. Ghosal, N. Mukherjee and A. K. Roy, Ann. Phys. (Berlin) 528, 796, (2016).

[37] K. Ch. Chatzisavvas, Ch. C. Moustakidis and C. P. Panos, J. Chem. Phys. 123, 174111 (2005).

[38] E. Romera and Á. Nagy, Phys. Lett. A 372, 4918 (2008).

[39] A. Grassi, Int. J. Quant. Chem. 108, 774 (2008).

[40] N. Flores-Gallegos, Chem. Phys. Lett. 666, 62 (2016).

[41] I. Varga and J. Pipek, Phys. Rev. E 68, 026202 (2003).

[42] R. Renner, N. Gisin and B. Kraus, Phys. Rev. A 72, 012332 (2005).

[43] P. Lévay, S. Negy and J. Pipek, Phys. Rev. A 72, 022302 (2005).

[44] F. Verstraete and J. I. Cirac, Phys. Rev. B 73, 094423 (2006).

[45] A. Bialas, W. Czyz and K. Zalewski, Phys. Rev. C 73, 034912 (2006).

[46] L. L. Salcedo, J. Math. Phys. 50, 012106 (2009).

[47] S.-B. Liu, C.-Y. Rong, Z.-M. Wu and T. Lu, Acta. Phys.-Chim. Sin. 31, 2057 (2015). 
[48] M. Gell-Mann and C. Tsallis (Eds.), Nonextensive Entropy-Interdisciplinary Applications, Chapter I, (Oxford University Press, 2004).

[49] J. Naudts, Generalised Thermostatistics, Spinger, London, 2011.

[50] A. R. Plastino and A. Plastino, Braz. J. Phys. 29, 79 (1999).

[51] J. Chen and G. Li, Entropy 16, 3009 (2014).

[52] Á. Nagy, Int. J. Quant. Chem. 115, 1392 (2015).

[53] Y. L. He, Y. Chen, J. N. Han, Z. B. Zhu, G. X. Xiang, H. D. Liu, B. H. Ma and D. C. He, Euro. Phys. J. D 69, 283 (2015).

[54] L. D. Site, Int. J. Quant. Chem. 115, 1396 (2015).

[55] D. R. Alcoba, A. Torre, L. Lain, G. E. Massaccesi, O. B. Oña, P. W. Ayers, M. V. Raemdonck, P. Bultinck and D. V. Neck, Theor. Chem. Acc. 135, 153 (2016).

[56] N. Flores-Gallegos, Chem. Phys. Lett. 650, 57 (2016).

[57] S. H. Patil, K. D. Sen, N. A. watson and H. E. Montgomery Jr., J. Phys. B 40, 2147 (2007).

[58] A. K. Roy, J. Phys. G 30, 269 (2004).

[59] K. D. Sen and A. K. Roy, Phys. Lett. A 357, 112 (2006).

[60] A. K. Roy, Int. J. Quant. Chem. 113, 1503 (2013); ibid., 114, 383 (2014).

[61] C. Tsallis, J. Stat. Phys. 52479 (1988). 


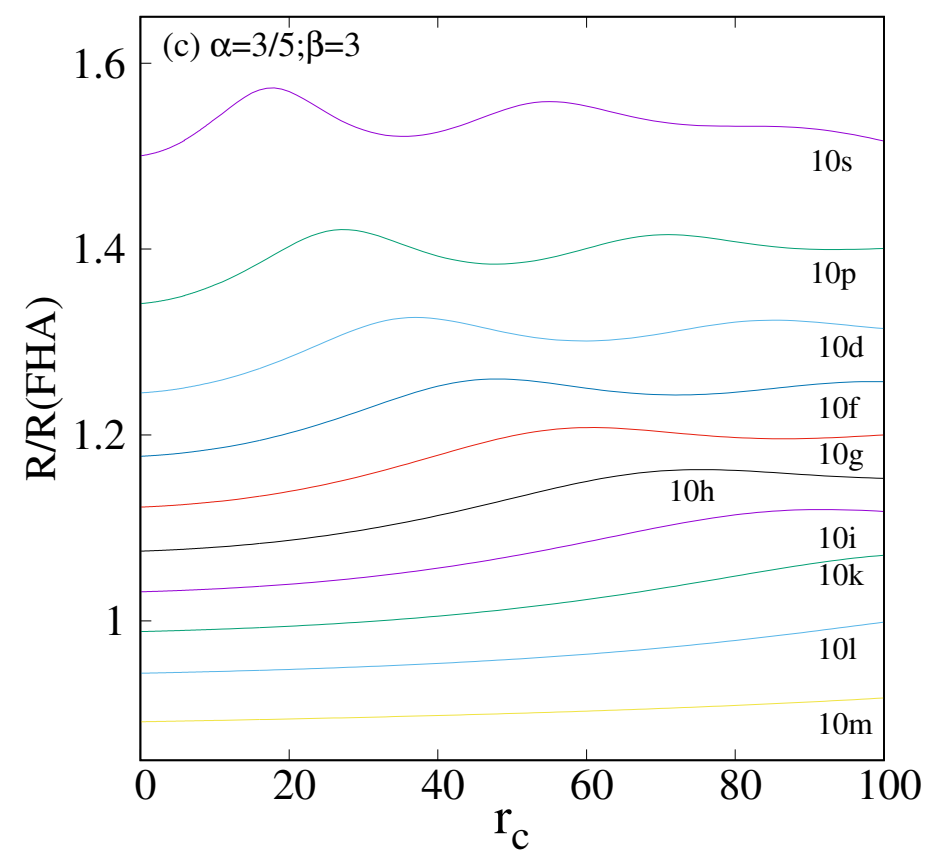




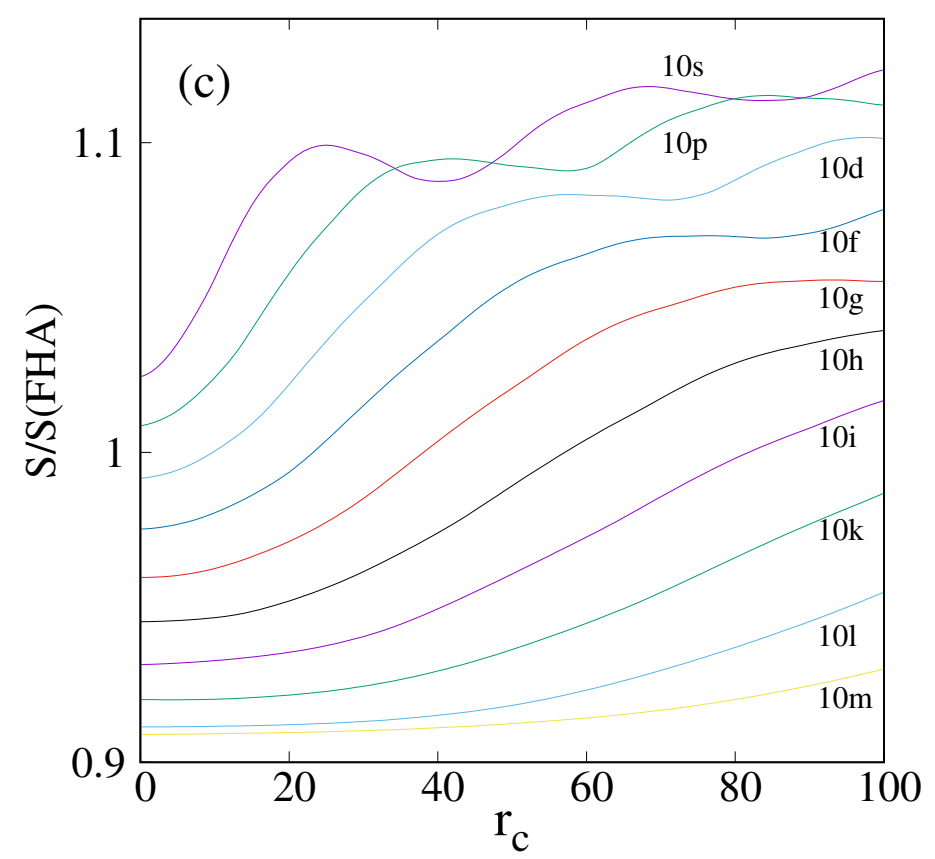




\section{Supplemental Materials: Information-entropic measures for non-zero $l$ states of confined hydrogen-like ions}

TABLE S1: Rényi entropies, $R_{\mathbf{r}}^{\alpha}, R_{\mathbf{p}}^{\beta}$ and $R_{t}^{(\alpha, \beta)}$ for $4 f, 5 g$ states of CHA at selected $r_{c}$ values, with $\alpha=\frac{3}{5}$ and $\beta=3$. See text for details.

\begin{tabular}{llll|llll}
\hline \hline$r_{c}$ & $R_{\mathbf{r}}^{\alpha}$ & $R_{\mathbf{p}}^{\beta}$ & $R_{t}^{(\alpha, \beta)}$ & $r_{c}$ & $R_{\mathbf{r}}^{\alpha}$ & $R_{\mathbf{p}}^{\beta}$ & $5 g^{\boldsymbol{q}}$ \\
\hline & & $4 f^{!}$ & & & & $R_{t}^{(\alpha, \beta)}$ \\
\hline 0.1 & -6.09881032904 & 13.5563109 & 7.4575006 & 0.1 & -6.09675536829 & 13.8239214 & 7.7271660 \\
0.2 & -4.01952824354 & 11.4774071 & 7.4578789 & 0.2 & -4.01733601542 & 11.7448104 & 7.7274744 \\
0.3 & -2.80329426627 & 10.2615533 & 7.4582590 & 0.3 & -2.80096355554 & 10.5287473 & 7.7277837 \\
0.5 & -1.27114583083 & 8.7301707 & 7.4590249 & 0.5 & -1.26853446452 & 8.9969400 & 7.7284055 \\
0.8 & 0.13835780349 & 7.3218298 & 7.4601876 & 0.8 & 0.14139955294 & 7.5879465 & 7.729346 \\
1 & 0.80744036310 & 6.6535319 & 7.4609723 & 1 & 0.81077543963 & 6.9192028 & 7.7299782 \\
7.5 & 6.83551829516 & 0.655708291 & 7.4912265862 & 7.5 & 6.85190763173 & 0.90113880 & 7.75304643 \\
15 & 8.87593577458 & -1.334419688 & 7.5415160866 & 25 & 10.42248270185 & -2.571994854 & 7.850487848 \\
75 & 11.39155328951 & -3.296164472 & 8.0953888175 & 95 & 12.54714379989 & -4.080092197 & 8.467051603 \\
100 & 11.39173125808 & -3.296184085 & 8.0955471731 & 100 & 12.54745436087 & -4.080121372 & 8.467332989 \\
\hline \hline
\end{tabular}

$R_{\mathbf{r}}^{\alpha}, R_{\mathbf{p}}^{\beta}, R_{t}^{(\alpha, \beta)}$ in FHA for $2 p$ states $(|m|=0)$ are: $11.391731416834988,-3.2961840848475106,8.095547331987477$.

${ }^{\top} R_{\mathbf{r}}^{\alpha}, R_{\mathbf{p}}^{\beta}, R_{t}^{(\alpha, \beta)}$ in FHA for $3 d$ states $(|m|=0)$ are: $12.547737094562322,-4.080150421410378,8.467586673151944$.

TABLE S2: Tsallis entropies $T_{\mathbf{r}}^{\alpha}, T_{\mathbf{p}}^{\beta}$ and $T_{t}^{(\alpha, \beta)}$ for $4 f, 5 g$ states of CHA at various $r_{c}$, for $\alpha, \beta$ as $\frac{3}{5}$ and 3 respectively. For more details, see text.

\begin{tabular}{llll|llll}
\hline \hline$r_{c}$ & $T_{\mathbf{r}}^{\alpha}$ & $T_{\mathbf{p}}^{\beta}$ & $T_{t}^{(\alpha, \beta)}$ & $r_{c}$ & $T_{\mathbf{r}}^{\alpha}$ & $T_{\mathbf{p}}^{\beta}$ & $T_{t}^{(\alpha, \beta)}$ \\
\hline & & $4 f^{!}$ & & & & $5 g^{\boldsymbol{q}}$ & \\
\hline 0.1 & -2.2819941539 & 0.4999999999991 & -1.140997077 & 0.1 & -2.281814882 & 0.4999999999995 & -1.140907441 \\
0.2 & -1.9991860306 & 0.4999999999463 & -0.9995930152 & 0.2 & -1.998746678 & 0.4999999999685 & -0.999373339 \\
0.3 & -1.6853746581 & 0.4999999993892 & -0.8426873280 & 0.3 & -1.684614841 & 0.4999999996420 & -0.842307420 \\
0.5 & -0.9964448564 & 0.4999999869370 & -0.4982224152 & 0.5 & -0.994873502 & 0.4999999923382 & -0.497436744 \\
0.8 & 0.1422579964 & 0.4999997815714 & 0.0711289671 & 0.8 & 0.1454747876 & 0.4999998717186 & 0.0727373752 \\
1 & 0.9530810038 & 0.4999991686466 & 0.4765397096 & 1 & 0.9576905936 & 0.4999995113174 & 0.4788448288 \\
7.5 & 35.9938331267 & 0.3652809476268 & 13.1478614733 & 7.5 & 36.247017479 & 0.4175385840553 & 15.134528355 \\
15 & 84.5658772343 & -6.7116097699 & -567.573167850 & 25 & 159.12580417 & -85.199117451 & -13557.37807917 \\
75 & 235.6526959759 & -364.23890302 & -85833.8794779 & 95 & 375.59604544 & -1748.9158539 & -656885.8785564 \\
100 & 235.6696500573 & -364.25321055 & -85843.4266642 & 100 & 375.64301711 & -1749.0179353 & -657006.3742152 \\
\hline \hline
\end{tabular}

$\overline{{ }^{!} T_{\mathbf{r}}^{\alpha}, T_{\mathbf{p}}^{\beta}, T_{t}^{(\alpha, \beta)} \text { in FHA for } 2 p \text { states }(|m|=0) \text { are: } 235.6696405610288,-364.25321044734767,-85843.4231793272 .}$

${ }^{\mathbf{I}} T_{\mathbf{r}}^{\alpha}, T_{\mathbf{p}}^{\beta}, T_{t}^{(\alpha, \beta)}$ in FHA for $3 d$ states $(|m|=0)$ are: $375.686384239036,-1749.1195832108906,-657120.411818189$. 
TABLE S3: $S_{\mathbf{r}}, S_{\mathbf{p}}$ and $S_{t}$ for $4 f, 5 g$ states of CHA at various $r_{c}$ values. See text for details.

\begin{tabular}{llll|llll}
\hline \hline$r_{c}$ & $S_{\mathbf{r}}$ & $S_{\mathbf{p}}$ & $S_{t}$ & $r_{c}$ & $S_{\mathbf{r}}$ & $S_{\mathbf{p}}$ & $S_{t}$ \\
\hline & & $4 f^{!}$ & & & & $5 g^{\boldsymbol{\top}}$ \\
\hline 0.1 & -6.3455584854 & 14.4653 & 8.1198 & 0.1 & -6.3503683331 & 14.8431 & 8.4928 \\
0.2 & -4.2663223577 & 12.3859 & 8.1196 & 0.2 & -4.2709619968 & 12.7637 & 8.4928 \\
0.3 & -3.0501348575 & 11.1695 & 8.1194 & 0.3 & -3.0546027165 & 11.5472 & 8.4926 \\
0.5 & -1.5180809495 & 9.6371 & 8.1191 & 0.5 & -1.5222004720 & 10.0147 & 8.4925 \\
0.8 & -0.1087231165 & 8.2272 & 8.1185 & 0.8 & -0.1123079582 & 8.6046 & 8.4923 \\
1 & 0.5602595052 & 7.5579 & 8.1181 & 1 & 0.5570394221 & 7.9351 & 8.4921 \\
7.5 & 6.5835558029 & 1.53173 & 8.11528 & 7.5 & 6.5968026117 & 1.89499 & 8.49179 \\
15 & 8.6122327603 & -0.460386 & 8.151846 & 25 & 10.1547840589 & -1.581652 & 8.573132 \\
75 & 10.8608517807 & -2.14839726 & 8.71245452 & 95 & 12.0497760380 & -2.86862362 & 9.18115241 \\
100 & 10.8608551968 & -2.14838651 & 8.71246868 & 100 & 12.0497927928 & -2.86859762 & 9.18119517 \\
\hline \hline
\end{tabular}

$!_{\mathbf{r}}, S_{\mathbf{p}}, S_{t}$ in FHA for $4 f$ states $(|m|=0)$ are: $10.8608551994597,-2.14838651125525,8.712468688204$.

${ }^{\Upsilon_{1}} S_{\mathbf{r}}, S_{\mathbf{p}}, S_{t}$ in FHA for $5 g$ states $(|m|=0)$ are: $12.0498008635736,-2.8685790066675,9.1812218569060$.

TABLE S4: $E_{\mathbf{r}}, E_{\mathbf{p}}, E_{t}$ for $4 f, 5 g$ states of CHA at various $r_{c}$. For more details, see text.

\begin{tabular}{llll|llll}
\hline \hline$r_{c}$ & $E_{\mathbf{r}}$ & $E_{\mathbf{p}}$ & $E_{t}$ & $r_{c}$ & $E_{\mathbf{r}}$ & $E_{\mathbf{p}}$ & $E_{t}$ \\
\hline & & $4 f^{!}$ & & & & $5 g^{\mathbf{I}}$ & \\
\hline 0.1 & 902.2306730117579 & 0.00000092547 & 0.0008349874 & 0.1 & 955.29622436210 & 0.00000066689 & 0.0006370775 \\
0.2 & 112.8081519710870 & 0.00000740021 & 0.000834804 & 0.2 & 119.41827435154 & 0.00000533361 & 0.0006369305 \\
0.3 & 33.4334274966356 & 0.00002496358 & 0.000834618 & 0.3 & 35.38507934013 & 0.00001799573 & 0.0006367803 \\
0.5 & 7.2254852493440 & 0.00011545852 & 0.0008342438 & 0.5 & 7.64401596722 & 0.00008326490 & 0.0006364782 \\
0.8 & 1.7654919808300 & 0.00047220577 & 0.0008336755 & 0.8 & 1.86653668200 & 0.00034074934 & 0.0006360211 \\
1 & 0.9044440955343 & 0.00092133060 & 0.000833292 & 1 & 0.95578172600 & 0.00066512447 & 0.0006357138 \\
7.5 & 0.0022027597662 & 0.37170465631 & 0.0008187761 & 7.5 & 0.00228068479 & 0.27382597673 & 0.0006245107 \\
15 & 0.0002938424484 & 2.71341384867 & 0.0007973162 & 25 & 0.00006598823 & 8.79992837948 & 0.0005806917 \\
75 & 0.0000384084606 & 17.97191418384 & 0.0006902736 & 95 & 0.00001189257 & 37.45698566645 & 0.0004454598 \\
100 & 0.000038408429927 & 17.97194614910 & 0.0006902742 & 100 & 0.00001189251 & 37.45679452927 & 0.0004454553 \\
\hline \hline
\end{tabular}

$!_{\mathbf{r}}, E_{\mathbf{p}}, E_{t}$ in FHA for $4 f$ states $(|m|=0)$ are: $0.000038408430432,17.97194626972933,0.00069027424804$.

${ }^{\top} E_{\mathbf{r}}, E_{\mathbf{p}}, E_{t}$ in FHA for $5 g$ states $(|m|=0)$ are: $0.0000118924912048,37.456994650567,0.000445456979443$. 